\title{
Competition of spinon Fermi surface and heavy Fermi liquid states from the periodic Anderson to the Hubbard model
}

\author{
Chuan Chen, ${ }^{1}$ Inti Sodemann ${ }^{1},{ }^{1, *}$ and Patrick A. Lee ${ }^{2, \dagger}$ \\ ${ }^{1}$ Max-Planck Institute for the Physics of Complex Systems, 01187 Dresden, Germany \\ ${ }^{2}$ Department of Physics, Massachusetts Institute of Technology, Cambridge, Massachusetts 02139, USA
}

(Received 14 October 2020; revised 4 February 2021; accepted 5 February 2021; published 19 February 2021)

\begin{abstract}
We study a model of correlated electrons coupled by tunneling to a layer of itinerant metallic electrons, which allows us to interpolate from a frustrated limit favorable to spin liquid states to a Kondo-lattice limit favorable to interlayer coherent heavy metallic states. We study the competition of the spinon Fermi-surface state and the interlayer coherent heavy Kondo metal that appears with increasing tunneling. Employing a slave rotor mean-field approach, we obtain a phase diagram and describe two regimes where the spin liquid state is destroyed by weak interlayer tunneling: (i) the Kondo limit in which the correlated electrons can be viewed as localized spin moments and (ii) near the Mott metal-insulator transition where the spinon Fermi surface transitions continuously into a Fermi liquid. We study the shape of local density of states (LDOS) spectra of the putative spin liquid layer in the heavy Fermi-liquid phase and describe the temperature dependence of its width arising from quasiparticle interactions and disorder effects throughout this phase diagram, in an effort to understand recent scanning tunneling microscopy experiments of the candidate spin liquid 1T-TaSe $\mathrm{T}_{2}$ residing on metallic $1 \mathrm{H}-\mathrm{TaSe}_{2}$. Comparison of the shape and temperature dependence of the theoretical and experimental LDOS suggests that this system is either close to the localized Kondo limit or in an intermediate coupling regime where the Kondo coupling and the Heisenberg exchange interaction are comparable.
\end{abstract}

DOI: 10.1103/PhysRevB.103.085128

\section{INTRODUCTION}

Since the pioneering proposal by Anderson [1-3], there has been an extensive quest to find quantum spin liquids (QSL) in materials [4-6]. Recently, it has been suggested that certain layered transition-metal dichalcogenide compounds might harbor a QSL state $[7,8]$. In particular, $1 \mathrm{~T}-\mathrm{TaS}_{2}$, a material that undergoes a commensurate charge density wave transition around $200 \mathrm{~K}$ into a $\sqrt{13} \times \sqrt{13}$ star of David structure $[9,10]$, remains insulating to the lowest temperatures in spite of having an odd number of electrons per star of David supercell, and yet shows no sign of any further conventional ordering phase transition such as antiferromagnetism that would double the unit cell, to the lowest measurable temperatures [11]. A possible connection to Anderson's proposal of a spin liquid was actually made from the very beginning [12], but somehow forgotten. The magnetic susceptibility of this compound remains nearly constant at low temperatures [13] and the material displays a finite linear in temperature specific-heat coefficient [14] indicative of a finite density of

\footnotetext{
*sodemann@pks.mpg.de

†palee@mit.edu
}

Published by the American Physical Society under the terms of the Creative Commons Attribution 4.0 International license. Further distribution of this work must maintain attribution to the author(s) and the published article's title, journal citation, and DOI. Open access publication funded by the Max Planck Society. states at low energies. Earlier experiments found no linear in temperature heat conductivity [15], which was taken as evidence against itinerant carriers. However, more recent experiments have shown a delicate sensitivity of heat transport to impurities [16], finding a finite linear in temperature heat conductivity in the cleanest samples. This indicates the presence of a finite density of states of itinerant carriers, as expected for the spinon Fermi-surface state. Moreover, band-structure analysis [17] showed that a single narrow band crosses the Fermi energy and is separated from other bands, making it very likely that the low-energy electronic behavior can be described by a single band Hubbard model.

A closely related compound, $1 \mathrm{~T}-\mathrm{TaSe}_{2}$, which also undergoes a commensurate charge density wave transition into the star of David structure, is expected to display similar phenomenology. While bulk 1T- $\mathrm{TaSe}_{2}$ is metallic [18], monolayer $1 \mathrm{~T}-\mathrm{TaSe}_{2}$ was studied by scanning tunneling microscopy (STM) and found to be a Mott insulator [19]. Recently Ruan et al. [20] extended their study by placing a monolayer of $1 \mathrm{~T}-\mathrm{TaSe}_{2}$ on top of a $1 \mathrm{H}-\mathrm{TaSe}_{2}$ monolayer, which is metallic. Surprisingly their experiment has found that a Kondo-like resonance peak near the Fermi energy develops in the tunneling density of states. It is important to emphasize that in these experiments the tunneling tip is coupled primarily to the originally insulating top layer of $1 \mathrm{~T}-\mathrm{TaSe}_{2}$. Therefore, taken at face value, the appearance of a tunneling densityof-states peak near zero bias may imply the destruction of the presumed spin liquid that would exist for $1 \mathrm{~T}-\mathrm{TaSe}_{2}$ in isolation and the formation of a coherent metallic state by the coupling with the substrate metallic $1 \mathrm{H}-\mathrm{TaSe}_{2}$, as it would 
be expected for the classic problem of Kondo heavy metal formation.

These experimental findings motivate us to consider a model consisting of a layer of correlated electrons coupled to a layer of noninteracting itinerant electrons via tunneling to study the competition of spinon Fermi-surface states and the heavy Kondo metals. There are two questions that we would like to address. First, the experimentalists found an excellent fit of the line shape and its temperature dependence with that expected for the Kondo resonance of a single impurity Kondo problem [20]. On the other hand, the actual system consists of a periodic array of local moments. Even if these are in the Kondo limit, the low-temperature state is expected to be a heavy fermion metal. Would the formation of a narrow coherent band lead to observable changes in the local density of states (LDOS)? Second, how does the Heisenberg exchange coupling $J_{H}$ between the local moments compete with the Kondo coupling $J_{K}$ that operates between the local moments and the conducting substrate? This problem was considered by Doniach [21] for the case when the Heisenberg coupling leads to an antiferromagnetic state. His conclusion is that the two relevant competing energy scales are the Kondo temperature $T_{K}$ and the Heisenberg exchange scale $J_{H}$. Note that at weak coupling $T_{K}$ is exponential small in terms of the Kondo coupling $J_{K}$. This would suggest that a very weak $J_{H}$ is sufficient to destroy the Kondo effect. If the experiment was interpreted as being in the Kondo limit, this places a rather small upper bound on $J_{H}$ of about $50 \mathrm{~K}$, since the scale $T_{K}$ is estimated to be about $50 \mathrm{~K}$ from the experimental fit [20]. With such a small Heisenberg coupling, the interpretation of the monolayer 1T-TaSe $\mathrm{T}_{2}$ as a spin liquid is brought into question. We note that the situation may change when the coupling becomes strong, and it may also change in frustrated spin models where the spin liquid state may be favored over the antiferromagnet. Notice that in the resonating valence bond picture, the quantum spin liquid is viewed as the superposition of a singlet formed between local moment pairs, while the Kondo phenomenon arises from the singlet formation between the local moment and the conduction-electron spin. The competition between different ways of forming singlets may well be different from the competition with an antiferromagnet considered by Doniach. With this in mind, we will consider a model that is sufficiently general to include the Hubbard interaction $(U)$ for the correlated electrons that reside in the putative spin liquid layer, which hop with an amplitude $\left(t_{d}\right)$ within this layer, and a tunneling amplitude $(V)$ to the itinerant electrons residing in the putative metallic layer, which hop with an amplitude $\left(t_{c}\right)$ within their own layer, as depicted in Fig. 1. This model therefore interpolates naturally between the periodic Anderson model $\left(t_{d} \rightarrow 0\right)$ where it would capture the physics of the formation of the interlayer coherent heavy Kondo metal [22,23] and the pure Hubbard limit $(V \rightarrow 0)$ where it would capture the traditional scenario for the appearance of the spinon Fermi-surface state near the Mott transition [24-26]. We note in passing that this model has been recently employed to understand angle-resolved photoemission spec-

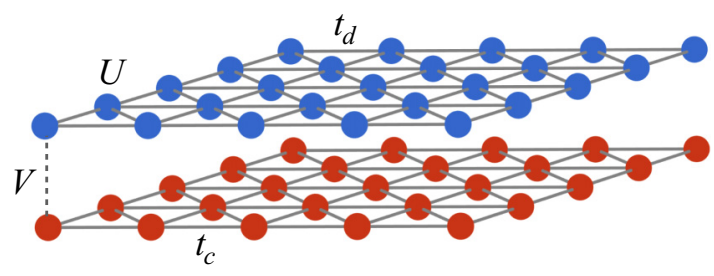

FIG. 1. Schematic of the model. The electrons in the top layer (blue) are correlated, with nearest-neighbor hopping $t_{d}$ and an on-site Hubbard interaction $U$. The bottom layer (red) hosts itinerant electrons with nearest-neighbor hopping $t_{c}$. There is also an interlayer tunneling $V$.

tra in $\mathrm{PdCrO}_{2}$ [27]; however, in this material the insulating layers are believed to be strong Mott insulators with $120^{\circ}$ spin antiferromagnetic order.

One of the central quantities of our interest will be the LDOS of the putative spin liquid layer, which is what has been measured in the aforementioned STM experiments. We are particularly interested in understanding the temperature dependence of the width of the LDOS peak, which can be used to try to learn about the microscopic parameters of the putative spin liquid and its coupling to the metal, and can guide us in determining where the system is likely to lie in the parameter space of our Hubbard-Anderson periodic model. Although an unambiguous quantitative description of the temperature dependence is challenging because it is controlled by the interplay of intrinsic quasiparticle lifetimes and extrinsic effects such as disorder induced broadening, we believe that our modeling is consistent with the system to be either close to the periodic Anderson model limit or in an intermediate coupling regime where the Kondo coupling and the Heisenberg exchange interaction are comparable, as we will discuss in detail. In the latter case, we cannot extract a tight bound on $J_{H}$ based on the experimental data.

Our paper is organized as follows: Sec. II sets up the model and describes the mean-field slave rotor approach that we employ to tackle it. Section III presents the solution of this mean field under a wide range of parameters, including not only the interplay between spinon Fermi surface and heavy metal but also the possibility of competing with Kondo insulating states. Section IV is devoted to a detailed analysis of the LDOS spectra and temperature dependence of the LDOS width and the comparison with STM experiments. Section V summarizes and further discusses our main findings. We have relegated some of the technical details of the mean-field treatment to Appendix A. In Appendix B we revisit the classic result of the temperature dependence of the single impurity Anderson model and give a more thorough derivation of the width of the Kondo resonance.

\section{MODEL AND SLAVE ROTOR APPROACH}

We consider a model of two-species of fermions residing in a triangular lattice that interpolates naturally between the Hubbard model and the periodic Anderson model. The 
microscopic Hamiltonian of the system has the form

$$
\begin{aligned}
H= & -t_{d} \sum_{\langle i, j\rangle, \sigma} d_{i, \sigma}^{\dagger} d_{j, \sigma}+\sum_{i} n_{d, i}\left(\epsilon_{d}^{(0)}-\mu_{F}\right) \\
& -t_{c} \sum_{\langle i, j\rangle, \sigma} c_{i, \sigma}^{\dagger} c_{j, \sigma}+\sum_{i} n_{c, i}\left(\epsilon_{c}^{(0)}-\mu_{F}\right) \\
& +\frac{U}{2} \sum_{i}\left(n_{d, i}-1\right)^{2}-V \sum_{i, \sigma}\left(c_{i, \sigma}^{\dagger} d_{i, \sigma}+\text { H.c. }\right)
\end{aligned}
$$

Here the electrons created by $c^{\dagger}$ are viewed as the "itinerant," and those created by $d^{\dagger}$ as the correlated ones. A schematic of the system is shown in Fig. 1. In the limit in which the correlated electrons are localized, $t_{d}=0$, this model reduces to the periodic Anderson model, and in the limit in which the two specifies are decoupled, $V=0$, the Hamiltonian for the correlated electrons reduces to the Hubbard model. We would like to employ a formalism capable of handling the various regimes of this model, and in particular the single occupancy constraints that appear in the large $U$ limit. For this purpose we resort to the slave rotor mean-field approach. According to the slave rotor method [24,28], the $d$ electron can be represented by bosonic rotor $\theta_{i}$ and fermionic spinon $f_{i, \sigma}$ degrees of freedom: $d_{i, \sigma} \equiv e^{i \theta_{i}} f_{i, \sigma}$, with the constraint $n_{\theta, i}+n_{f, i}=1$. The Hamiltonian can be then written in terms of these partons as follows:

$$
\begin{aligned}
H= & -t_{d} \sum_{\langle i, j\rangle, \sigma} e^{-i \theta_{i}} e^{i \theta_{j}} f_{i, \sigma}^{\dagger} f_{j, \sigma}+\sum_{i} n_{f, i}\left(\epsilon_{d}^{(0)}-\mu_{F}\right) \\
& -t_{c} \sum_{\langle i, j\rangle, \sigma} c_{i, \sigma}^{\dagger} c_{j, \sigma}+\sum_{i} n_{c, i}\left(\epsilon_{c}^{(0)}-\mu_{F}\right) \\
& +\frac{U}{2} \sum_{i} n_{\theta, i}^{2}-V \sum_{i, \sigma}\left(e^{i \theta_{i}} c_{i, \sigma}^{\dagger} f_{i, \sigma}+\text { H.c. }\right) .
\end{aligned}
$$

\section{A. Mean-field theory}

In the spirit of a mean-field theory we approximate the ground state of Eq. (2) by a direct product of a rotor state and a spinon state. The constraint on the rotor and spinon occupation is satisfied on average:

$$
\left\langle n_{\theta, i}\right\rangle+\left\langle n_{f, i}\right\rangle=1 .
$$

Since the rotor and spinon degrees of freedom are assumed to be disentangled, we write the mean-field Hamiltonian as the sum of a rotor part and a fermionic part, i.e., $H_{\mathrm{MF}}=H_{f}+H_{\theta}$, with

$$
\begin{aligned}
H_{f}= & -T_{f} \sum_{\langle i, j\rangle, \sigma} f_{i, \sigma}^{\dagger} f_{j, \sigma}+\sum_{i} n_{f, i}\left(\epsilon_{d}^{(0)}+\lambda-\mu_{F}\right) \\
& -t_{c} \sum_{\langle i, j\rangle, \sigma} c_{i, \sigma}^{\dagger} c_{j, \sigma}+\sum_{i} n_{c, i}\left(\epsilon_{c}^{(0)}-\mu_{F}\right) \\
& -V_{f} \sum_{i, \sigma} c_{i, \sigma}^{\dagger} f_{i, \sigma}+\text { H.c. } \\
H_{\theta}= & -2 \sum_{\langle i, j\rangle} T_{\theta} e^{-i \theta_{i}} e^{i \theta_{j}}+\sum_{i} \frac{U}{2} n_{\theta, i}^{2}+\lambda n_{\theta, i}-4 V_{\theta} \cos \left(\theta_{i}\right), \\
T_{f}= & t_{d}\left\langle e^{-i \theta_{i}} e^{i \theta_{j}}\right\rangle_{\theta},
\end{aligned}
$$

$$
\begin{aligned}
& V_{f}=V\left\langle e^{i \theta_{i}}\right\rangle_{\theta}, \\
& T_{\theta}=t_{d}\left\langle f_{i, \sigma}^{\dagger} f_{j, \sigma}\right\rangle_{f}, \\
& V_{\theta}=V\left\langle c_{i, \sigma}^{\dagger} f_{i, \sigma}\right\rangle_{f},
\end{aligned}
$$

where a Lagrange multiplier $\lambda$ is introduced to maintain the constraint Eq. (3). The quasiparticle residue of the correlated $d$ electron is $\left\langle e^{i \theta_{i}}\right\rangle \equiv \Phi$. This can be regarded as the order parameter for the metallic phase: when it is nonzero there will be a coherent tunneling between the spinon and itinerant electrons. In this paper, we will concentrate on the competition of this correlated metallic state and a more exotic state, known as the spinon Fermi-surface state, that arises when $\Phi=0$ and the spinon, $f$, has a Fermi surface.

We expect that the essence of the competition between these phases does not depend substantially on the details of the fermion dispersions, and therefore, in order to simplify analytical treatment, we will approximate the band structure for spinons $(f)$ and itinerant electrons $(c)$ by simple parabolic bands:

$$
\begin{aligned}
H_{f} & =\sum_{k, \sigma} f_{k, \sigma}^{\dagger} f_{k, \sigma} \epsilon_{f, k}+c_{k, \sigma}^{\dagger} c_{k, \sigma} \epsilon_{c, k}-V_{f}\left(c_{k, \sigma}^{\dagger} f_{k, \sigma}+\text { H.c. }\right), \\
\epsilon_{f, k} & =\frac{3}{2} T_{f}\left(k^{2}-\frac{\Lambda^{2}}{2}\right)+\lambda-\mu_{F}, \\
\epsilon_{c, k} & =\frac{3}{2} t_{c}\left(k^{2}-\xi \frac{\Lambda^{2}}{2}\right)-\mu_{F}
\end{aligned}
$$

where $\Lambda$ is a cutoff on $k$ space intended to mimic the finite size of the Brillouin zone which can be determined by equaling $\pi \Lambda^{2}$ to the area of the triangular lattice's Brillouin zone, and the lattice constant $a_{0}$ is taken to be 1 . The dimensionless parameter $\xi$ in $\epsilon_{c, k}$ reflects the occupancy of $c$ electrons when $c$ and $f$ fermions are decoupled (since in such case $\lambda=0$ and $\mu_{F}=0$, see discussions in the following section): the number of $c$ electrons per site is $\xi$ when the dispersion $\epsilon_{c, k}$ is particlelike $\left(t_{c}>0\right)$, and $2-\xi$ with holelike dispersion $\left(t_{c}<0\right)$. See Fig. 2 for a schematic illustration.

\section{B. Expectation values of the rotor operators}

Notice that even after the mean-field decoupling, the rotor Hamiltonian $H_{\theta}$ is still essentially a two-dimensional quantum $\mathrm{XY}$ model with a transverse field which is not amenable to analytic treatment. Therefore, one has to make further approximations.

We are interested in solutions that respect time-reversal and translational symmetry and that have no flux per unit cell. Therefore we seek for self-consistent solutions where $\Phi$ is uniform and real. To do so, we perform an additional self-consistent mean-field treatment of $H_{\theta}$ by introducing an effective single-site rotor Hamiltonian:

$$
\begin{aligned}
H_{\theta}^{(1)} & =-K_{\theta}\left(e^{i \theta}+e^{-i \theta}\right)+\frac{U}{2} n_{\theta}^{2}+\lambda n_{\theta}, \\
K_{\theta} & =2 z T_{\theta} \Phi+2 V_{\theta},
\end{aligned}
$$

with $z$ being the lattice coordination $(z=6$ for the triangular lattice). To lowest order in perturbation theory in $K_{\theta} / U[\lambda=0$ 


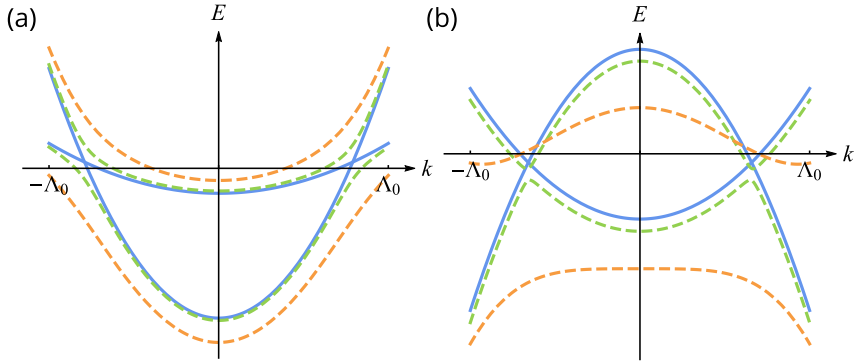

FIG. 2. Schematic of the band dispersion. (a) Particle-particle dispersion (with $\xi>1$ ). Blue solid lines indicate the $\epsilon_{f, k}$ and $\epsilon_{c, k}$ in the spin liquid phase; green dashed lines stand for the $E_{1, k}$ and $E_{2, k}$ for small $V_{f}$, where both bands cross the Fermi level and there are two Fermi surfaces; the orange dashed lines are for when $V_{f}$ is large such that the $E_{2, k}$ band is fully occupied and $E_{1, k}$ is partly occupied to maintain the half filling of the spinon. (b) Particle-hole dispersion $(\xi<1)$. For small $V_{f}$ (green dashed line) only $E_{1, k}$ crosses the Fermi level and has two Fermi surfaces while the $E_{2, k}$ is fully occupied; when $V_{f}$ is large enough (orange dashed lines) there is only one Fermi surface.

since we are interested in the half-filled spinon and the constraint Eq. (3) leads to $\left\langle n_{\theta, i}\right\rangle=0$ ] we have $\Phi=4 K_{\theta} / U$. On the other hand, in the opposite limit in which $K_{\theta} / U \gg 1$, we have $\theta \approx 0$ and thus $\Phi=\left\langle e^{i \theta}\right\rangle=1$. Moreover, since $\Phi=\left\langle e^{i \theta}\right\rangle$ is never greater than 1 , we introduce the following natural interpolation between these limits:

$$
\Phi=\frac{K_{\theta}}{\sqrt{(U / 4)^{2}+K_{\theta}^{2}}},
$$

or, equivalently,

$$
K_{\theta}=\frac{U}{4} \frac{\left\langle e^{i \theta}\right\rangle}{\sqrt{1-\left\langle e^{i \theta}\right\rangle^{2}}} .
$$

Although the above mean-field treatment captures well the behavior of the residue $\Phi$, it ignores completely the nearestneighbor rotor correlations, which are essential in order to obtain a dispersion for the spinon. To capture these, and since $V_{\theta}$ is small near the metal to insulator phase transition, we will approximate their value by performing a perturbative calculation directly with the more complete rotor Hamiltonian $H_{\theta}$ from Eq. (4b), which contains the $U$ and $T_{\theta}$ terms only,

$$
\tilde{H}_{\theta}=\frac{U}{2} \sum_{i} n_{\theta, i}^{2}-2 T_{\theta} \sum_{\langle i, j\rangle} e^{-i \theta_{i}} e^{i \theta_{j}}
$$

which leads to the following nearest-neighbor rotor correlations:

$$
\left\langle e^{-i \theta_{i}} e^{i \theta_{j}}\right\rangle \approx \frac{4 T_{\theta}}{U}
$$

where it should be noted that these nearest-neighbor rotor correlations from Eq. (10) are needed to reproduce the spinon bandwidth which is expected to be given by the Heisenberg exchange coupling scale $J_{H}=4 t_{d}^{2} / U$. The expressions above are all zero-temperature results. The finite temperature versions of these formulas are discussed in Appendix A.

\section{Expectation values of the fermion operators}

The fermionic mean-field Hamiltonian is free from interactions and can be diagonalized exactly. Because we are already accounting for spinon hopping in the spin liquid phase at $V=0$, the correlator $\left\langle f_{i, \sigma}^{\dagger} f_{j, \sigma}\right\rangle$ is not expected to change much during the spin liquid to heavy metal phase transition, so we will simply approximate its value when $c$ and $f$ fermions are decoupled from each other $\left(V_{f}=0\right.$ in the insulating phase):

$$
\left\langle f_{i, \sigma}^{\dagger} f_{j, \sigma}\right\rangle=\frac{1}{N} \sum_{k} e^{i \vec{k} \cdot \vec{\delta}} n_{F}\left(\epsilon_{f, k}\right) \equiv \chi_{0},
$$

where $n_{F}$ is the Fermi-Dirac distribution function $n_{F}(x)=$ $1 /\left(e^{\beta x}+1\right), \delta$ is the distance between sites $i$ and $j$, and $N$ is the total number of lattice sites in Eq. (11). Thus $T_{\theta}=t_{d} \chi_{0}$. As for the hybridization between the itinerant electrons and spinons, one obtains

$$
\begin{aligned}
\left\langle c_{i, \sigma}^{\dagger} f_{i, \sigma}\right\rangle & =V_{f} \chi_{c f}, \\
\chi_{c f} & =-\frac{1}{2 N} \sum_{k} \frac{n_{F}\left(E_{1, k}\right)-n_{F}\left(E_{2, k}\right)}{\sqrt{\left(\frac{\epsilon_{f, k}-\epsilon_{c, k}}{2}\right)^{2}+V_{f}^{2}}}
\end{aligned}
$$

It should be noted that Eq. (12a) is an exact result of solving the free fermionic Hamiltonian $H_{f}$, although in the $V_{f} \rightarrow 0$ limit the $\chi_{c f}$ reduces to the $c$ - $f$ hybridization susceptibility of the $c$ - $f$ decoupled Hamiltonian. The quasiparticle energy dispersions read (see Fig. 2)

$$
E_{1 / 2, k}=\frac{\epsilon_{f, k}+\epsilon_{c, k}}{2} \pm \sqrt{\left(\frac{\epsilon_{f, k}-\epsilon_{c, k}}{2}\right)^{2}+V_{f}^{2}},
$$

and the occupancy of the spinon reads

$$
\begin{aligned}
& \left\langle f_{i, \sigma}^{\dagger} f_{i, \sigma}\right\rangle=\frac{1}{N} \sum_{k} \cos ^{2}\left(\alpha_{k}\right) n_{F}\left(E_{1, k}\right)+\sin ^{2}\left(\alpha_{k}\right) n_{F}\left(E_{2, k}\right) \\
& \cos \left(2 \alpha_{k}\right)=\frac{\epsilon_{f, k}-\epsilon_{c, k}}{2} / \sqrt{\left(\frac{\epsilon_{f, k}-\epsilon_{c, k}}{2}\right)^{2}+V_{f}^{2}}
\end{aligned}
$$

\section{Self-consistent equations}

Once the expressions for the expectation values of the rotor and fermions are obtained, the self-consistent equations for the order parameter $\Phi$ can be derived; from Eqs. (6b), (8), and (12a), one can show that

$$
\frac{\Phi}{8}\left(\frac{1}{\sqrt{1-\Phi^{2}}}-8 z \frac{t_{d}}{U} \chi_{0}\right)=\Phi \frac{V^{2}}{U} \chi_{c f} .
$$

Therefore, one needs to solve Eq. (15) along with the constraint Eq. (3) and $\left\langle n_{f, i}\right\rangle=1$. Equation (15) always has a trivial solution $\Phi=\left\langle e^{i \theta_{i}}\right\rangle=0$, and the nontrivial solution of $\left\langle e^{i \theta_{i}}\right\rangle$ satisfies

$$
\frac{1}{8}\left(\frac{1}{\sqrt{1-\Phi^{2}}}-8 z \frac{t_{d}}{U} \chi_{0}\right)=\frac{V^{2}}{U} \chi_{c f} .
$$

It should be noted that the "susceptibility" $\chi_{c f}$ also depends on $\Phi$, through its dependence on $V_{f}$ in Eq. (12b), which in turn depends on $\Phi$ via Eq. (4d). 


\section{MEAN-FIELD PHASE DIAGRAM AND MEAN-FIELD PROPERTIES}

To explore the phase transition between the spin liquid and heavy metal phases, it is important to distinguish the cases with the band dispersions of the $d$ electron and itinerant electrons being particle-particle like $\left(t_{d}>0\right.$ and $\left.t_{c}>0\right)$ and particle-hole like $\left(t_{d}>0\right.$ and $\left.t_{c}<0\right)$. Here we discuss in detail the behavior when the itinerant fermion has higher density (larger Fermi-surface area) than the spinon, which is most relevant to the recent experiments $1 \mathrm{~T}-\mathrm{TaS}_{2}$ and $1 \mathrm{~T}-\mathrm{TaSe}_{2}$. Namely, we will take the parameter $\xi$, that controls the density of the itinerant electrons in Eq. (5c), to have a range of $1 \leqslant \xi<2$ for the particle-particle case and $0 \leqslant \xi<1$ for the particle-hole case (this leads to $n_{c} \geqslant n_{f}$ in the insulating phase; see Fig. 2 for an illustration).

\section{A. Particle-particle dispersion}

In this section we discuss the situation for particleparticle-like dispersions. As mentioned before, there are two competing phases in our phase diagram: the spin liquid phase and the heavy metal phase (see Fig. 5 for an example of the phase diagram). The phases are determined by whether order parameter $\Phi$ is finite (heavy metal) or zero (spin liquid). When $t_{d} \sim 0$, the model reduces to a periodic Anderson model and the transition from spin liquid to heavy metal is of the form of a weak-coupling instability. On the other hand, for larger $t_{d} / U \sim 1 / 8$ and $V=0$, the system exhibits a metal-insulator (Mott) transition, as one expects from a Hubbard model. The goal of the next section is to determine how the phase boundary evolves between these two regimes.

\section{Phase boundary}

The phase boundary is obtained when $\Phi=0$ is a solution of Eq. (16). According to the constraint from Eq. (3) and $\left\langle n_{f, i}\right\rangle=1$, we have that $\left\langle n_{\theta, i}\right\rangle=0$. This leads to a value $\lambda=0$ for the Lagrange multiplier in Eq. (4b). Thus one just needs to self-consistently adjust the chemical potential $\mu_{F}$ such that the spinon is half filled. Along the phase boundary, since $c$ and $f$ fermions are decoupled, this can be satisfied by setting $\mu_{F}=0$, which leads to $n_{f, i}=1$ and $n_{c, i}=\xi$, which corresponds to two Fermi surfaces from the two bands with Fermi momentum $k_{F, f}=\Lambda / \sqrt{2}$ and $k_{F, c}=\Lambda \sqrt{\xi / 2}$. In this case the susceptibility of $c-f$ coupling from Eq. (12b) reduces to

$$
\begin{aligned}
\chi_{c f}^{(0)} & =-\frac{1}{N} \sum_{k} \frac{n_{F}\left(\epsilon_{f, k}\right)-n_{F}\left(\epsilon_{c, k}\right)}{\epsilon_{f, k}-\epsilon_{c, k}} \\
& =\frac{1}{\Lambda^{2}} \frac{2}{3} \frac{1}{T_{f}-t_{c}} \ln \left(\frac{T_{f}}{t_{c}}\right) .
\end{aligned}
$$

It is interesting to notice that the $\chi_{c f}^{(0)}$ is independent of $\xi$, in other words, the density of itinerant electrons. This implies that the phase boundary is insensitive to the $c$ electron's density within the parabolic band approximation. The critical value at which the residue $\Phi$ and the hybridization between the itinerant and correlated electron, $V_{f}$, become simultane- (a)

$V_{c}^{2} /\left(U \cdot t_{c}\right)$

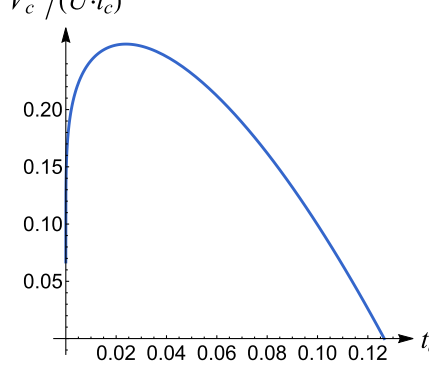

FIG. 3. (a) Phase boundary between spin liquid (below blue curve) and heavy metal with particle-particle dispersion and $\xi=1.2$. As $t_{d} \rightarrow 0$, the critical coupling $V_{c}^{2} / U$ is suppressed logarithmically with $t_{d} / U$; when $V=0$ (horizontal axis), the metal-insulator transition occurs at $t_{d} / U \sim 1 / 8$. Near this critical point, the $V_{c}^{2} / U$ has a linear dependence on $t_{d} / U$. (b) Plot of the $\chi_{c f}$ with $T_{f}=0.1 t_{c} . \chi_{c f}$ saturates at small $V_{f}$, while for $V_{f}>V_{f}^{*}$, it is a decreasing function of $V_{f}$.

ously nonzero is given by

$$
\frac{V_{c}^{2}}{U t_{c}}=\frac{1}{8}\left(1-8 z \frac{t_{d}}{U} \chi_{0}\right)\left(\frac{4 t_{d}^{2} \chi_{0}}{U t_{c}}-1\right) \frac{\frac{3}{2} \Lambda^{2}}{\ln \left(\frac{4 t_{d}^{2} \chi_{0}}{t_{c} U}\right)} .
$$

A plot of the phase boundary in this case can be found in Fig. 3(a). As it approaches the Anderson $\left(t_{d} \rightarrow 0\right)$ limit, the critical $V_{c}^{2} / U$ has a logarithmic dependence on $t_{d} / U$. This means that in the local moment limit, the heavy Fermiliquid phase is destabilized by a weak Heisenberg coupling, $J_{H} \sim t_{d}^{2} / U$, comparable to the Kondo scale, $T_{K} \sim \rho^{-1} e^{-1 / J_{K} \rho}$ (with $J_{K} \sim V^{2} / U$ and $\rho^{-1} \sim t_{c}$ ). This is responsible for the sharp narrowing of the region of the heavy Fermi-liquid phase in the local moment limit $V^{2} \ll t_{c} U$, and $t_{d} \ll U$, as shown in Fig. 3(a). Around the axis $V=0$ we recover the physics of the spin liquid to metal (Mott) transition in the conventional Hubbard model with the spin liquid to metal transition (see [24]) occurring at $t_{d} / U=1 /\left(8 z \chi_{0}\right)$, which in the case of the triangular lattice corresponds to $t_{d} / U \sim 1 / 8$ and is in line with previous cluster mean-field calculation [28].

\section{Turning on of the heavy fermion phase}

As one enters the heavy fermion metallic phase ( $\Phi$ becomes finite), both the $E_{1, k}$ and $E_{2, k}$ bands cross the Fermi level [as indicated by the green dashed lines in Fig. 2(a)]. According to Eq. (14a), the spinon density in this case is

$$
\left\langle f_{i, \sigma}^{\dagger} f_{i, \sigma}\right\rangle=\frac{k_{F 1}^{2}+k_{F 2}^{2}}{2 \Lambda^{2}}+\frac{\sum_{\alpha=c, f} \epsilon_{\alpha, k_{F 1}}+\epsilon_{\alpha, k_{F 2}}}{3 \Lambda^{2}\left(t_{c}-T_{f}\right)},
$$

and by requiring this to be $1 / 2$ one can obtain $\mu_{F}=0$ (with $\lambda=0$ ). It can be shown that in this case the susceptibility is simply a constant:

$$
\chi_{c f}=\frac{2}{3} \frac{1}{t_{c} \Lambda^{2}} \frac{1}{\frac{4 t_{d}^{2} \chi_{0}}{U t_{c}}-1} \ln \left(\frac{4 t_{d}^{2} \chi_{0}}{U t_{c}}\right) .
$$

Notice that $\chi_{c f}$ is independent of $V_{f}$ (or $\Phi$ ), which is a consequence of the parabolic model. Physically $\chi_{c f}$ should be a monotonically decreasing function of $V_{f}$ for a general 
band dispersion, but we conclude from the above that it is weakly dependent on these parameters whenever the bands can be approximated by parabolas. Nevertheless, Eq. (20) still unveils an important effect of the correlated fermion hopping $t_{d}$, which is to set a "cutoff" to $\chi_{c f}$, as depicted in Fig. 3(b). Such cutoff would otherwise be absent in the pure periodic Anderson model $\left(t_{d} \rightarrow 0\right)$ and we would have that $\chi_{c f} \rightarrow \infty$ as $V_{f} \rightarrow 0$. This divergence is responsible for the weak-coupling (Kondo) instability of the periodic Anderson model that leads to the formation of the heavy Fermi-liquid state.

On the other hand, there is a further phase transition that appears within the heavy Fermi-liquid state, associated with the disappearance of one of the Fermi surfaces while preserving the net Luttinger volume, at large $V_{f}$. This occurs when $V_{f}$ is larger than some critical value $V_{f}^{*}=\frac{3}{2} \frac{\Lambda^{2}}{2} \sqrt{T_{f} t_{c}(2-\xi)}$, for which we have that $E_{2, \Lambda}<0$, so the $E_{2, k}$ band is fully occupied and there is only one Fermi surface associated with the band $E_{1, k}$ [see yellow dashed lines in Fig. 2(a)]. In this case, the density of the spinon reads

$$
\begin{aligned}
\left\langle f_{i, \sigma}^{\dagger} f_{i, \sigma}\right\rangle= & \frac{k_{F 1}^{2}+\Lambda^{2}}{2 \Lambda^{2}} \\
& +\frac{\epsilon_{f, k_{F 1}}+\epsilon_{c, k_{F 1}}+\sqrt{\left(\epsilon_{f, \Lambda}-\epsilon_{c, \Lambda}\right)^{2}+4 V_{f}^{2}}}{3 \Lambda^{2}\left(t_{c}-T_{f}\right)},
\end{aligned}
$$

and the $\mu_{F}$ can be determined by requiring $\left\langle f_{i, \sigma}^{\dagger} f_{i, \sigma}\right\rangle=1 / 2$. In this case the susceptibility $\chi_{c f}$ is no longer independent of $V_{f}$ (we do not show the explicit expression here since it is too lengthy). Figure 3(b) shows a plot of the $\chi_{c f}$ as a function of $V_{f}$ for a specific parametrization. As mentioned before, a finite $t_{d}$ sets a cutoff to the $\chi_{c f}$; moreover, the critical $V_{f}^{*}$ will also decrease as $t_{d}$ decreases. This role of $t_{d}$ as a cutoff of the $\chi_{c f}$ susceptibility leads to an increasing value of the critical $V$ as $t_{d}$ increases at extremely small values of $t_{d}$, as shown in Fig. 3(a). In other words, the larger the value of $t_{d}$ the smaller the susceptibility to induce the mixing between the itinerant and correlated fermions.

However, the physical role of $t_{d}$ is not exclusively to cut off $\chi_{c f}$. It is clear from Fig. 3(a) that at sufficiently large $t_{d}$ the critical $V$ starts to decrease as $t_{d}$ increases. The other physical role of $t_{d}$ can be understood from the self-consistent equation for the residue $\Phi, \mathrm{Eq}$. (16), where we see that the hopping of correlated electrons $t_{d}$ appears not only inside $\chi_{c f}$, but also on the left-hand side of the equation, arising from the coupling between nearest-neighbor rotors in $H_{\theta}\left(t_{d} e^{-i \theta_{i}} e^{i \theta_{j}}\right)$. This term competes with the interaction part $\left(\approx U n_{\theta, i}^{2}\right)$ and tends to "lock" the angles of nearby rotors; therefore, in this second role, $t_{d}$ tends to enhance the appearance of a residue and therefore favors the destruction of the spin liquid in favor of the appearance of the finite $\Phi$ leading to a metallic state.

To illustrate more concretely these contrasting roles of $t_{d}$ we compare the solution of $\Phi$ as a function of $V^{2} / U$ for different types of modified self-consistent equations. As shown by the dashed curves in Fig. 4, when the susceptibility $\chi_{c f}$ is replaced by one which diverges logarithmically at small $V_{f}$ (dashed lines), there is always a weak-coupling instability to the heavy fermion phase, while for the exact $\chi_{c f}$ (solid lines) one has to reach a finite critical value of $V$ for the occurrence

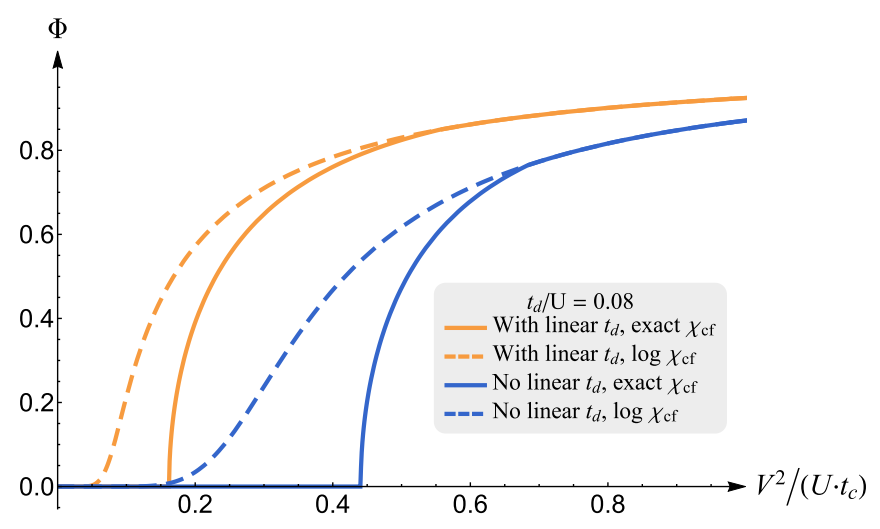

FIG. 4. Solution of $\Phi$ for different types of self-consistent equations. The orange lines stand for the self-consistent equation with the linear $t_{d}$ (nearest-neighbor coupling) term while the blue lines are for the case without the linear $t_{d}$ term. The solid lines are for the case with the exact form of $\chi_{c f}$ with a cutoff while the dashed curves stand for the case with a (logarithmically) diverging $\chi_{c f}$ at small $V_{f}$. The logarithmically diverging $\chi_{c f}$ always supports a weak-coupling instability to the heavy metal phase while for the exact $\chi_{c f}$ there is a threshold of $V$ for the onset metallic phase. The linear $t_{d}$ term in the left-hand side of the self-consistent equation will also help boost the heavy fermion phase, as expected.

of the heavy metal phase. Moreover, when the linear $t_{d}$ term from the left-hand side of Eq. (16) is removed (blue lines), the heavy metal phase is also suppressed and one needs a larger $V$ to get a nonzero $\Phi$.

From the analysis above, one can see that either a very large $t_{d}$ (nearby rotors lock strongly) or a very small $t_{d}$ (susceptibility of the $c-f$ coupling diverges) will enhance the tendency towards heavy Fermi-liquid order and suppress the tendency towards the spin liquid insulating phase. This conclusion is further confirmed by the (zero-temperature) phase diagram Fig. 5 obtained by explicitly solving the selfconsistent equation [the boundary in this phase diagram is the same previously shown in Fig. 3(a)]. As can be seen from Fig. 5, the insulating spin liquid phase has a dome shape in the phase diagram, which will be suppressed by very small or large $t_{d}$. The gray dashed line indicates the critical value of $V$, above which the $E_{2}$ band is fully occupied and the metallic phase has a single Fermi surface. The orange dashed line marks the boundary where the two heavy fermion bands start to develop an indirect gap, which occurs for parameters above such orange line (see further discussion in Sec. IV).

\section{B. Particle-hole dispersion}

In this section we discuss the results for the case where itinerant electrons are holelike which can be accounted for by simply changing $t_{c} \rightarrow-t_{c}$ in their energy dispersion [Eq. (5c)].

\section{Phase boundary}

When the metallic electron's band structure is holelike, the susceptibility $\chi_{c f}$ will have a stronger $\xi$ dependence compared to the particle-particle case. It can be shown that within the 


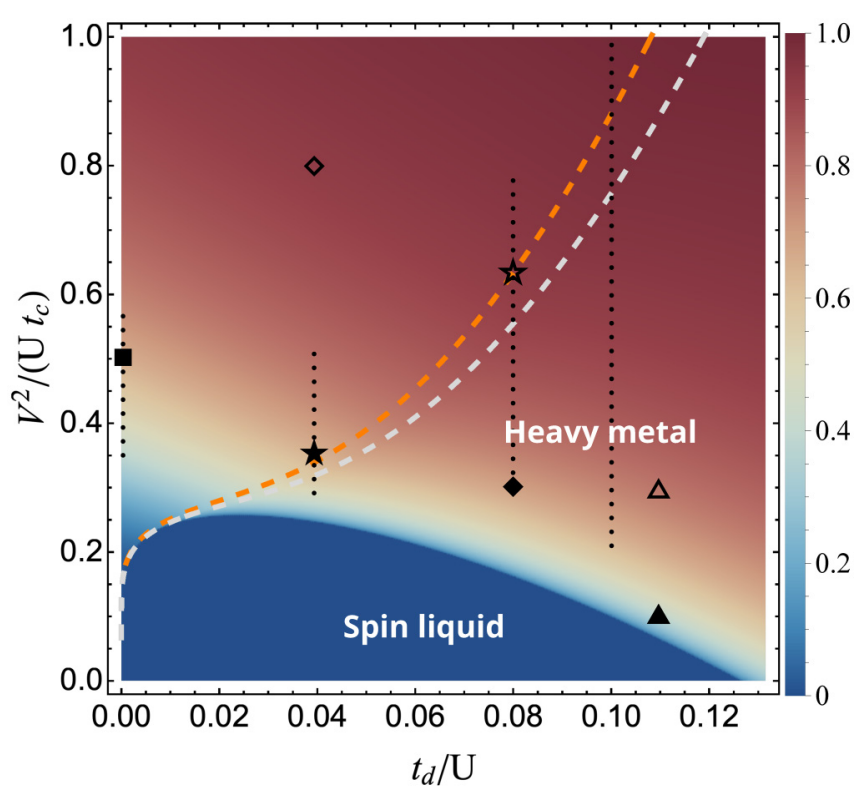

FIG. 5. Phase diagram with $\xi=1.2$ (density plot of $\Phi$ ). The vertical scale is proportional to the Kondo coupling scale $J_{K} \sim V^{2} / U$ while the horizontal scale is proportional to the hopping between the correlated electrons. The dark blue region is the spin liquid with $\Phi=0$ and the light blue and red region stand for the heavy metal phase. The gray dashed curve is the critical value of $V$ where the number of Fermi surfaces of the system changes from 2 (below) to 1 (above) and the $\chi_{c f}$ changes from a constant plateau to a monotonically decreasing function of $V_{f}$ [see Fig. 3(b)]. The orange dashed curve indicates where the two heavy quasiparticle bands develop an indirect band gap. Dotted lines and symbols indicate where detailed LDOS spectra are calculated as a guiding reference for subsequent Figs. 11-14.

spin liquid phase $\left(V_{f}=0\right)$ it is given by

$$
\chi_{c f}^{(0)}=\frac{2}{3 \Lambda^{2}\left(T_{f}+t_{c}\right)} \ln \left(\frac{\left(T_{f} / t_{c}+\xi\right)\left(T_{f} / t_{c}+2-\xi\right)}{T_{f} / t_{c}(1-\xi)^{2}}\right)
$$

Thus for $\xi=1$, i.e., when both the itinerant electrons and spinons are at half filling, the two bands are perfectly nested, and the band structure leads to a divergent susceptibility $\chi_{c f}$ for all values of $t_{d}$, which indicates that the spin liquid is unstable against a transition into the Kondo insulating phase at arbitrarily small $V$. Figure 6(a) shows the phase boundary between the spin liquid and the heavy fermion metallic phase. Similar to the particle-particle case, as $t_{d} \rightarrow 0$, the critical value of $J_{K} \sim V^{2} / U$ decreases logarithmically with $t_{d}$. Moreover, for the particle-hole case, the phase boundary now also has a $\xi$ dependence, as expected from the $\xi$ dependence of $\chi_{c f}^{(0)}$. As $\xi \rightarrow 1$, the spin liquid phase is suppressed, and when $\xi=1$ it only exists along the $V=0$ line [Fig. 6(a)]. It should be noted that at $V=0$, the critical $t_{d} / U$ for the Mott transition is always the same "universal" value around $1 / 8$; this is because the $d$ and $c$ electrons are decoupled in this case and the problem reduces to the metal to insulator transition for the triangular lattice Hubbard model. (a)

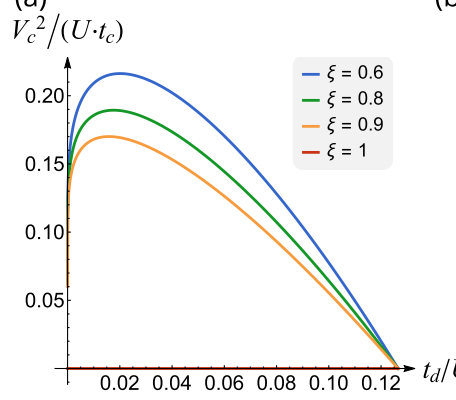

(b)

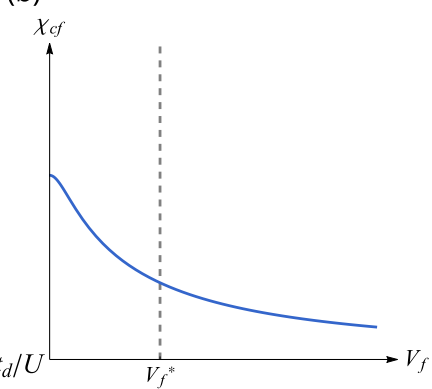

FIG. 6. (a) Phase boundary for particle-hole dispersion at various filling of the metallic electrons. As $\xi \rightarrow 1$, the spin liquid phase gets suppressed and at exactly half filling of the metal it can exist only within the $V=0$ line. (b) $\chi_{c f}$ as a function of $V_{f}$ for the particle-hole dispersion with $\xi=0.6, T_{f}=0.1 t_{c}$. Similar to the particle-particle case, $\chi_{c f}$ is a decreasing function of $V_{f}$.

\section{Turning on of the heavy fermion phase}

For the case with $\xi<1$, weakly inside the heavy fermion metallic phase, where the quasiparticles' energy dispersions $E_{1, k}$ and $E_{2, k}$ have the Mexican-hat shape, it turns out that in order to maintain the half-filling constraint of the spinon, we find that the $E_{2, k}$ band is fully filled while the $E_{1, k}$ band is partially occupied and features two Fermi surfaces, as shown by the green dashed lines in Fig. 2(b). The $\mu_{F}$ can be solved from $\left\langle f_{i, \sigma}^{\dagger} f_{i, \sigma}\right\rangle=1 / 2$ and the $\chi_{c f}$ as a function of $V_{f}$ can be obtained accordingly. Similar to the particle-particle case, at finite $t_{d}, \chi_{c f}$ tends to saturate as $V_{f} \rightarrow 0$ and it is diverging in the atomic limit $\left(t_{d} \rightarrow 0\right)$. For rather large $V_{f}, E_{1, \Lambda}$ becomes smaller than zero and there is only one Fermi surface for the system [see the orange dashed lines in Fig. 2(b)]. A plot of $\chi_{c f}$ at $\xi=0.6$ is shown in Fig. 6(b); as expected, it is a decreasing function of $V_{f}$. The phase diagram for this case is shown in Fig. 7.

As for the special case when $\xi=1$, as explained before, because the spinon and the itinerant electron bands are nested in this case, the susceptibility $\chi_{c f}$ diverges as $V_{f} \rightarrow 0$. As a result, one expects a weak-coupling instability from the spin liquid state to that with heavy electrons. Notice, however, that this state is not a metal but a Kondo insulator, since the Fermi surfaces are completely gapped out by the hybridization due to the perfect nesting. As can be seen from Fig. 8, the Kondo insulating phase turns on more rapidly for larger $t_{d} / U$. The phase diagram for this case is shown in Fig. 9.

\section{TUNNELING DOS}

In the recent experiment by Ruan et al. [20], a monolayer $1 \mathrm{~T}-\mathrm{TaSe}_{2}$, which is originally an insulator, is placed on top of a metallic monolayer $1 \mathrm{H}-\mathrm{TaSe}_{2}$. The system was studied by STM, where the tip is primarily coupled to the top layer (1T-TaSe 2 ). Surprisingly, a narrow peak around zero bias was found. It was found that this coherent peak can be broadened by increasing temperature and the temperature dependence of its width can be fitted to a form [see Eq. (28)] which describes the Kondo resonance for the single impurity Kondo problem [as shown in Fig. 2(c) of [20]]. This observation was then taken as an indication of the existence of the local magnetic 


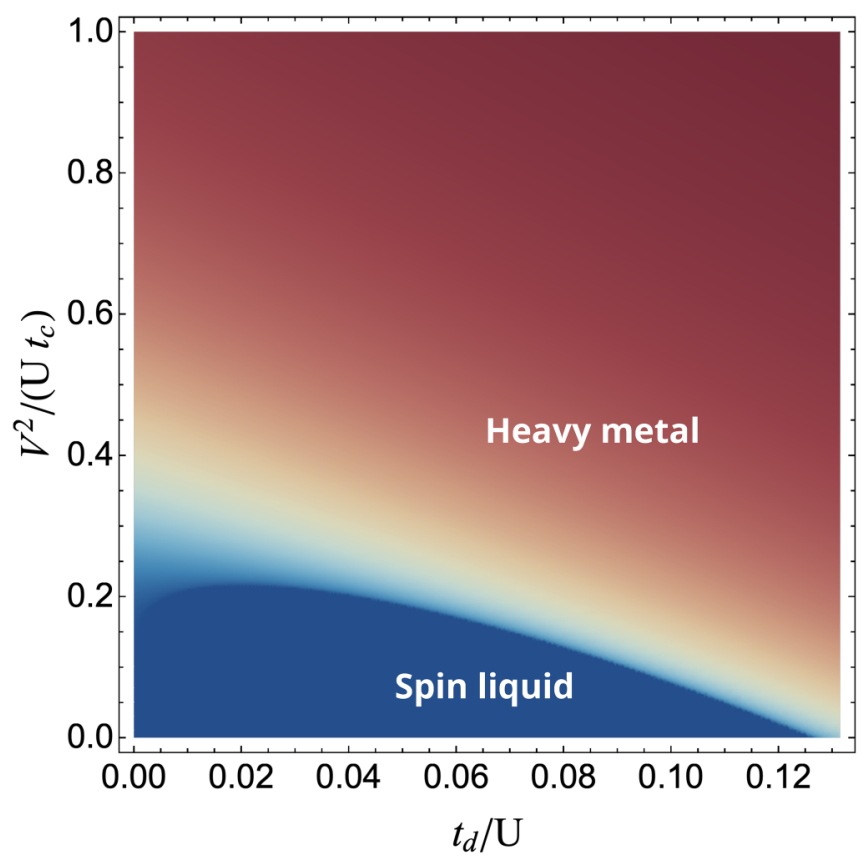

FIG. 7. Phase diagram for the particle-hole case with $\xi=0.6$. The spin liquid phase has a dome shape and the phase boundary has qualitatively the same behavior as the particle-particle case.

moment in the $1 \mathrm{~T}-\mathrm{TaSe}_{2}$ layer, which couples to the metallic substrate (the $1 \mathrm{H}$ layer). Combining this with the further observation of a real-space modulation of the electronic structure, it was suggested that the pristine $1 \mathrm{~T}-\mathrm{TaSe}_{2}$ monolayer is likely to host the QSL phase.

This motivates us to study if this behavior could also appear in our theoretical model, e.g., in certain regimes of the heavy metal phase. In this section, we discuss the behavior of the LDOS of the correlated $d$ electrons in the metallic phase, which is the quantity reflected by the STM $d I / d V$ curve. The thermal Green function of the $d$ electron can be written as

$$
\begin{aligned}
G_{d}(\tau, \mathbf{r}) & =-\left\langle T_{\tau} d_{\mathbf{R}+\mathbf{r}}(\tau) d_{\mathbf{R}}^{\dagger}(0)\right\rangle \\
& =G_{f}(\tau, \mathbf{r}) G_{\theta}(\tau, \mathbf{r}),
\end{aligned}
$$

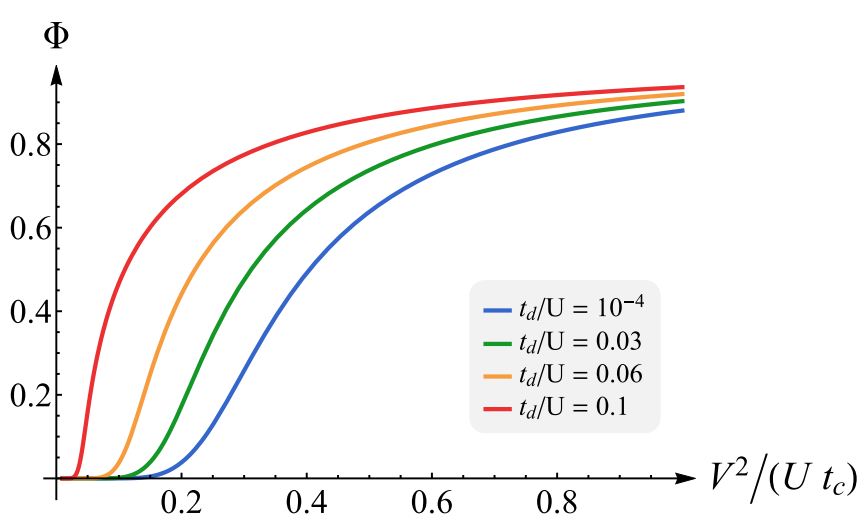

FIG. 8. $\Phi$ as a function of $V^{2} / U$ for $\xi=1$ at different values of $t_{d} / U$. As expected, the metallic phase turns on in the form of a weakcoupling instability with $V$.

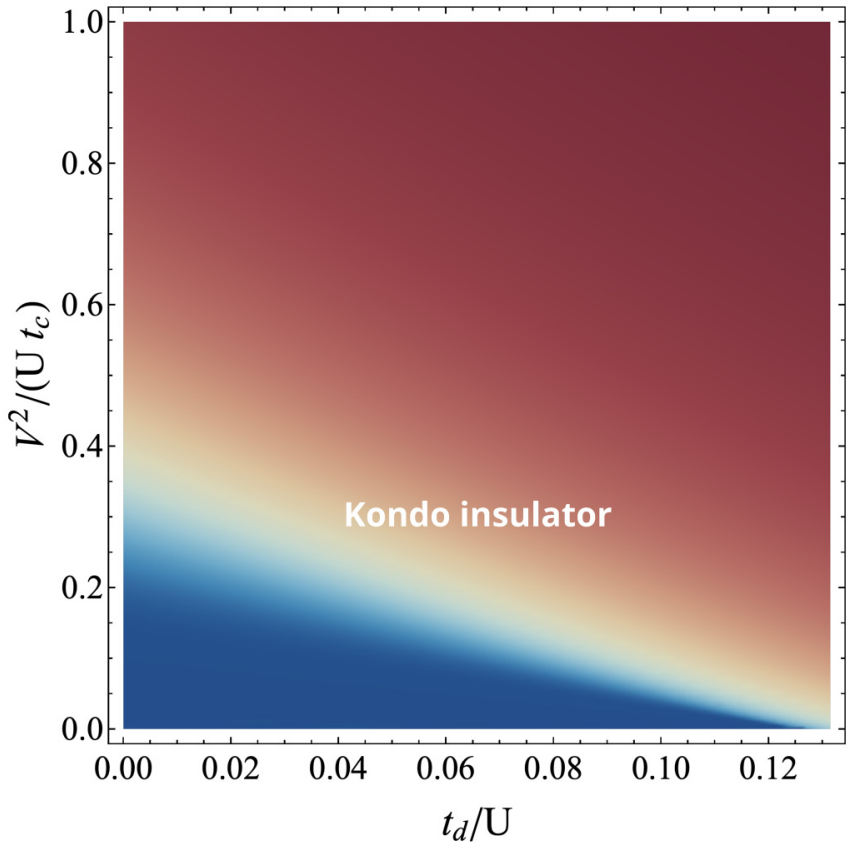

FIG. 9. Phase diagram for the particle-hole case with perfect nesting $(\xi=1)$. The system is Kondo insulating at any finite $V$ since the Fermi surfaces of the heavy electrons are fully gapped out, and the spin liquid phase exists strictly only at the $V=0$ line.

where $G_{f}(\tau, \mathbf{r})$ and $G_{\theta}(\tau, \mathbf{r})$ are Green functions of the spinon and rotor, with the definition

$$
\begin{aligned}
& G_{f}(\tau, \mathbf{r})=-\left\langle T_{\tau} f_{\mathbf{R}+\mathbf{r}}(\tau) f_{\mathbf{R}}^{\dagger}(0)\right\rangle, \\
& G_{\theta}(\tau, \mathbf{r})=\left\langle T_{\tau} e^{i \theta_{\mathbf{R}+\mathbf{r}}(\tau)} e^{-i \theta_{\mathbf{R}}(0)}\right\rangle .
\end{aligned}
$$

As pointed out from previous studies [24,28], the Matsubara Green function of $d$ electrons can be separated into a coherent part and an incoherent part:

$$
\begin{aligned}
G_{d}\left(i \omega_{n}, \mathbf{r}\right) & =G_{d}^{\mathrm{coh}}\left(i \omega_{n}, \mathbf{r}\right)+G_{d}^{\mathrm{inc}}\left(i \omega_{n}, \mathbf{r}\right), \\
G_{d}^{\mathrm{coh}}\left(i \omega_{n}, \mathbf{r}\right) & =\Phi^{2} G_{f}\left(i \omega_{n}, \mathbf{r}\right) .
\end{aligned}
$$

The coherent part is mainly peaked at $\omega \sim 0$ while the incoherent part captures features at larger energy scales $\omega \sim U$. In this paper, we are mainly interested in the feature of LDOS near $\omega=0$ and we will focus on the coherent part. From the slave rotor mean-field theory, since the fermionic part of the Hamiltonian is noninteracting, it can be shown that the Matsubara Green function of the spinon has the form

$$
G_{f}\left(i \omega_{n}, k\right)=\cos ^{2}\left(\alpha_{k}\right) G_{1}\left(i \omega_{n}, k\right)+\sin ^{2}\left(\alpha_{k}\right) G_{2}\left(i \omega_{n}, k\right),
$$

where $G_{1 / 2}\left(i \omega_{n}, k\right)=1 /\left(i \omega_{n}-E_{1 / 2, k}\right)$ is the Green function of the self-consistent band-diagonal quasiparticles that result from the coherent mixing of the correlated and the itinerant electron. By analytical continuation, the spectral function of the spinons can be obtained:

$$
\begin{aligned}
A_{f}(\omega, k) & =-\frac{1}{\pi} \operatorname{Im} G_{f}\left(\omega+i 0_{+}, k\right) \\
& =\cos ^{2}\left(\alpha_{k}\right) \delta\left(\omega-E_{1, k}\right)+\sin ^{2}\left(\alpha_{k}\right) \delta\left(\omega-E_{2, k}\right),
\end{aligned}
$$



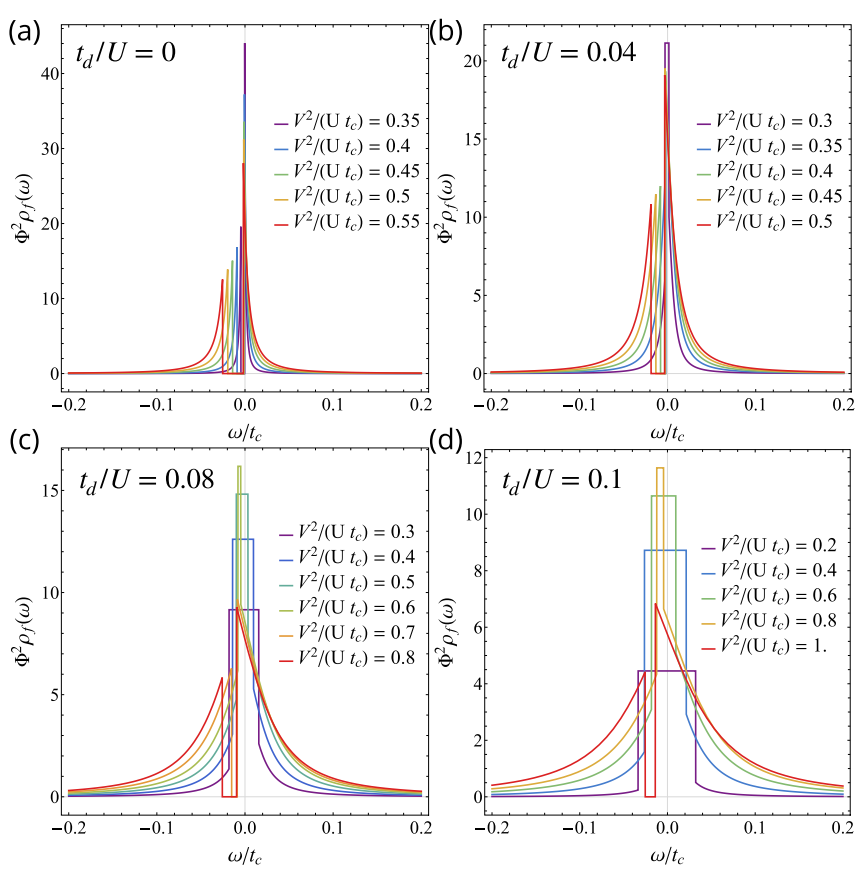

FIG. 10. Mean-field LDOS without disorder and quasiparticle lifetime broadening effects for the case of (a) $t_{d} / U=0$, (b) $t_{d} / U=0.04$, (c) $t_{d} / U=0.08$, and (d) $t_{d} / U=0.1$. Within each case, the Kondo coupling $J_{K} \sim V^{2} / U$ is increased gradually (along the black dotted lines in Fig. 5). In the Anderson limit, it is clear that within the heavy metal phase, there is a coherent gap opened below the Fermi level. On the other hand, when $t_{d} / U$ is finite, the spinon band is dispersive with a finite bandwidth. So for small $J_{K}$, the band dispersions of heavy quasiparticles are still overlapping with each other [see the green dashed lines in Fig. 2(a)], and lead to a plateaulike LDOS at small $\omega$. When $J_{K}$ is large and above the orange dashed line in the phase diagram (see Fig. 5), the two heavy quasiparticle bands are fully separated in energy and the LDOS exhibits a gap between the two peaks.

and the LDOS for the spinon $\rho_{f}(\omega)=\frac{1}{N} \sum_{k} A_{f}(\omega, k)$ can be obtained accordingly.

\section{A. Zero-temperature mean-field LDOS}

We are particularly interested in understanding the tunneling density of states for experiments in $1 \mathrm{~T}-\mathrm{TaSe}_{2}$ where the dispersion of the itinerant electron is likely to be particlelike. Here we explored in detail the particle-particle case and we take the bare band filling of the itinerant electrons to be $\xi=1.2$ (this value is taken arbitrarily as the physics should not be very sensitive to the detailed value of $\xi$ ). We are mainly focused on three regimes: (i) the Anderson limit with $t_{d}=0$, (ii) moderate $t_{d}$ along the orange dashed line in Fig. 5, and (iii) large $t_{d}$ near the metal-insulator transition of the Hubbard model.

Figure 10 shows the zero-temperature mean-field LDOS of correlated $d$ electrons at different regimes of the phase diagram, as indicated by the black dotted lines in Fig. 5. In the Anderson limit [see Fig. 10(a)], the mean-field LDOS opens a coherent band gap enhanced by increasing the Kondo coupling $J_{K}$, which is the expected behavior for the periodic Anderson model. When $t_{d} / U$ is finite [see Figs. 10(b)-10(d)], the spinon acquires a band dispersion. Consequently, when $\Phi$ is small at small $J_{K}$, the quasiparticle bands are still overlapping with each other in energy [see green dashed line in Fig. 2(a)] and the LDOS shows a plateaulike peak near $\omega \sim 0$. The width of the plateau is given mainly by the spinon bandwidth. As $J_{K}$ becomes larger, the overlap between the two bands decreases and the width of the flat peak is reduced. At some intermediate scale marked by the orange dashed line in Fig. 5, the Kondo coupling and the Heisenberg exchange interaction compete, resulting in a narrow peak the width of which is much less than $J_{K}$ or $J_{H}$ individually. Finally, when $J_{K}$ is greater than a critical value indicated by the orange dashed line in Fig. 5, the two quasiparticle bands become fully separated and the LDOS behaves similarly to the Anderson limit with a finite gap sandwiched by two peaks. As can be seen clearly, near the metal-insulator transition of the Hubbard model, the LDOS peak is much broader than in the small $t_{d} / U$ limit. It should be noted that the perfect flatness of the peak is an artifact of parabolic band dispersion adopted in our paper, and a more realistic tight-binding model would give rise to a dispersive peak. Below we will describe how these LDOS features are broadened by temperature and by extrinsic disorder effects.

\section{B. Broadening due to finite temperature and disorder}

At finite temperature the tunneling conductance is given by the LDOS convolved with the thermal broadening due to the thermal distribution of electrons in the lead. This effect has been removed in the experiment [29] and we also do not include it in our theory. After removing this, it is notable that the experiment shows a single peak which can be fitted with a Lorentzian with a temperature-dependent half-maximum half width:

$$
\Gamma_{\exp }=\sqrt{2 T_{K}^{2}+\pi^{2} T^{2}} .
$$

This form of the width was found in an earlier experiment that detected the Kondo peak in a single impurity and has been considered a signature of the single impurity Kondo problem [29]. The low-temperature width therefore allows us to extract $T_{K}$ from experiments. Furthermore, at large temperatures compared to $T_{K}$ the width scales approximately as $\pi T$, which places a constraint on the theory. We have reexamined the theoretical basis of Eq. (28) and came to the conclusion that while the derivation given in [29] is not well justified and there is a small correction to the width from Eq. (28) at low temperatures, it does provide a correct value of the slope of the $\Gamma-T$ curve at high temperatures, which is $\pi$. Details are given in Appendix B. In this paper we do not fit the experimental data to the single impurity Kondo problem, but rather to the periodic Anderson-Hubbard model. As we shall see below, by introducing a Fermi-liquid-type quasiparticle lifetime together with a disorder induced width, it is possible to fit the data in certain parameter ranges.

As it is well known from the theory of single Kondo impurity and Kondo lattice problems [30-33], the fluctuations around the mean-field configuration which give rise to quasiparticle interactions lead to a characteristic temperature- and frequency-dependent quasiparticle lifetime. In order to account for these effects, we add the following 
semiphenomenological imaginary part to the quasiparticle self-energy [34]:

$$
\Sigma_{\mathrm{FL}}(\omega, T)=-i \frac{1}{2 \pi E_{0}}\left[\omega^{2}+\left(\pi k_{B} T\right)^{2}\right] .
$$

In addition to this intrinsic quasiparticle interaction lifetime, disorder is another important agent in broadening the density of states in experiments, and we account for this by adding a constant impurity scattering rate $\gamma_{0}$ into the imaginary part of the self-energy, as follows:

$$
\begin{aligned}
G_{1 / 2}\left(\omega+i 0_{+}, k\right) & =\frac{1}{\omega-E_{1 / 2, k}-\Sigma(\omega, T)}, \\
\Sigma(\omega, T) & =-i \gamma_{0}+\Sigma_{\mathrm{FL}}(\omega, T) .
\end{aligned}
$$

It should be noted that the energy scale $E_{0}$ controlling the quasiparticle interaction effects in Eq. (29) is usually of the order of the bandwidth for a normal Fermi liquid (large $t_{d}$ ), while for a Kondo lattice $\left(t_{d}=0\right)$, it is of the order of the Kondo temperature $T_{K} \sim 2 V_{f}^{2} / D_{c}$ with $D_{c}$ being the half bandwidth of itinerant electrons. In order to capture both regimes, we use a phenomenological expression of $E_{0}$ that interpolates between these two limits, as follows:

$$
E_{0}=\sqrt{T_{K}^{2}+W_{\mathrm{sp}}^{2}}
$$

with $W_{\mathrm{sp}}$ being the spinon bandwidth.

As mentioned above, in the Anderson limit, the mean-field LDOS will have two peaks separated by the gap. However, once the self-energy is included, the mean-field spectral function will be broadened and it is possible to obtain a single-peak behavior. This can be seen clearly from Fig. 11, which shows the case of $t_{d} / U=0, V^{2} / U=0.5 t_{c}$ (as indicated by the $\mathbf{~ i n}$ Fig. 5). By including only the $\Sigma_{\text {FL }}$ [see Fig. 11(a)], at very low temperatures, the LDOS has two peaks separated by a band gap. When a finite impurity scattering rate (here we take $\gamma_{0}=0.05 t_{c}$ ) is taken into account, the LDOS is broadened into a single peak, as shown in Fig. 11(b). We further calculated the half-maximum half width of LDOS at different temperatures and compare it with the experimental results. We fit our theoretical data with a function of the form

$$
\Gamma=\sqrt{\left(\Gamma_{0}\right)^{2}+a \pi^{2}\left(k_{B} T\right)^{2}},
$$

which is expected for the single-impurity Anderson model $[35,36]$. Previous theoretical works find that the experimental data can be well fitted with $a \approx 1$. According to our theoretical calculation, for the case with $V^{2} / U=0.5 t_{c}$ and $\gamma_{0}=0.05 t_{c}$, the data can be well fitted with $a \approx 0.85$, as can be seen from Fig. 11(c), where all quantities are presented in unit of $t_{c}$. Nevertheless, once we take $t_{c}=105 \mathrm{meV}$ so that the lowesttemperature width matches with the experimental one, we also find quantitatively good fit to the experimental result. In other words, the experimental data can be described by a periodic Anderson model with a finite impurity scattering rate.

When $t_{d}$ is finite, as shown in the mean-field results above, one expects to see either a plateaulike peak (with small $J_{K}$ ) or a finite gap sandwiched by two peaks (rather large $J_{K}$ ) in the LDOS. In any case, the inclusion of a finite imaginary self-energy can broaden the curve. Along the orange line, since the two mean-field bands of heavy quasiparticles
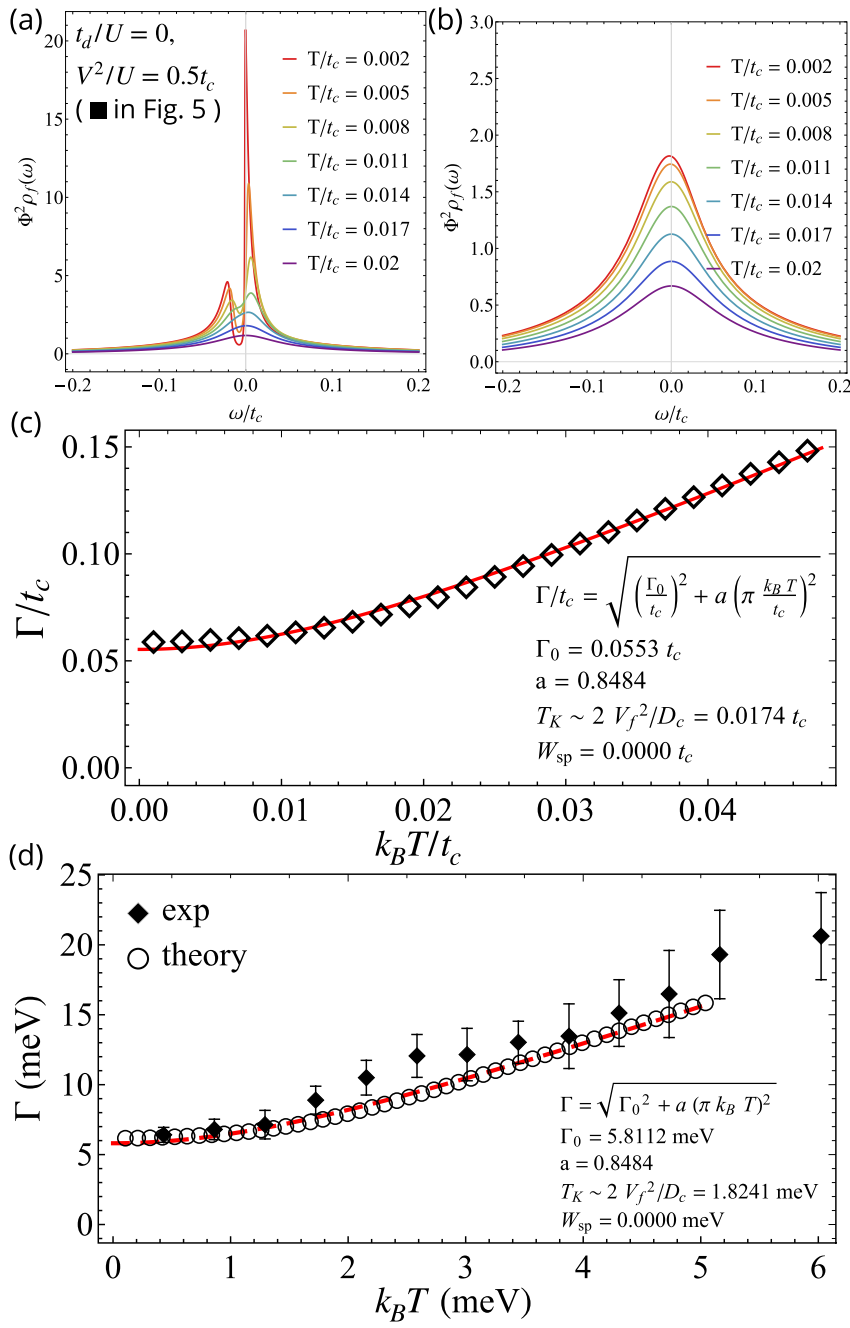

FIG. 11. LDOS for the particle-particle case $(\xi=1.2)$ with $t_{d} / U=0, V^{2} / U=0.5 t_{c}$ (indicated by $\boldsymbol{\square}$ in Fig. 5). (a) LDOS with the self-energy being $\Sigma_{\mathrm{FL}}(\omega, T)$ only. It is clear that in the lowtemperature limit, the spectral function has the two-peak behavior at $\omega \sim 0$, which is due to the opening of a band gap in the dispersion of heavy quasiparticles. This is the signature of a coherent heavy fermion band in the Kondo lattice problem. At higher temperature, there is only a single peak around $\omega \sim 0$ due to the broadening effects in $\Sigma_{\mathrm{FL}}(\omega, T)$. (b) LDOS for self-energy from Eq. (30b) with $\gamma_{0}=0.05 t_{c}$. In this case the disorder effect $\left(\gamma_{0}\right.$ term) is able to broaden the LDOS and changes it into a single peak. (c) Width in units of $t_{c}$. (d) Fitting to experimental data (extracted from [20]) with $t_{c}=105 \mathrm{meV}$. The experimental data can be well fitted by the theoretical result.

are about to separate, the LDOS of the spinon should have only a single peak around $\omega \sim 0$. Figures 12 (a) -12 (c) and 13(a)-13(c) show two points close to the line: $t_{d} / U=0.04$, $V^{2} / U=0.35 t_{c}$ and $t_{d} / U=0.08, V^{2} / U=0.65 t_{c}$ (indicated by $\star$ and $\lesssim$, respectively, in Fig. 5); it is clear that the LDOS has only a single peak at $\omega \sim 0$. We find that the width as a function of temperature can also be relatively well fitted by Eq. (32). To compare with the experimental data, as we did for the Anderson limit, one can tune $t_{c}$ so that at the lowest temperatures the width is consistent with the experimental one. Figures 12(c) and 13(c) show the comparison of the width 


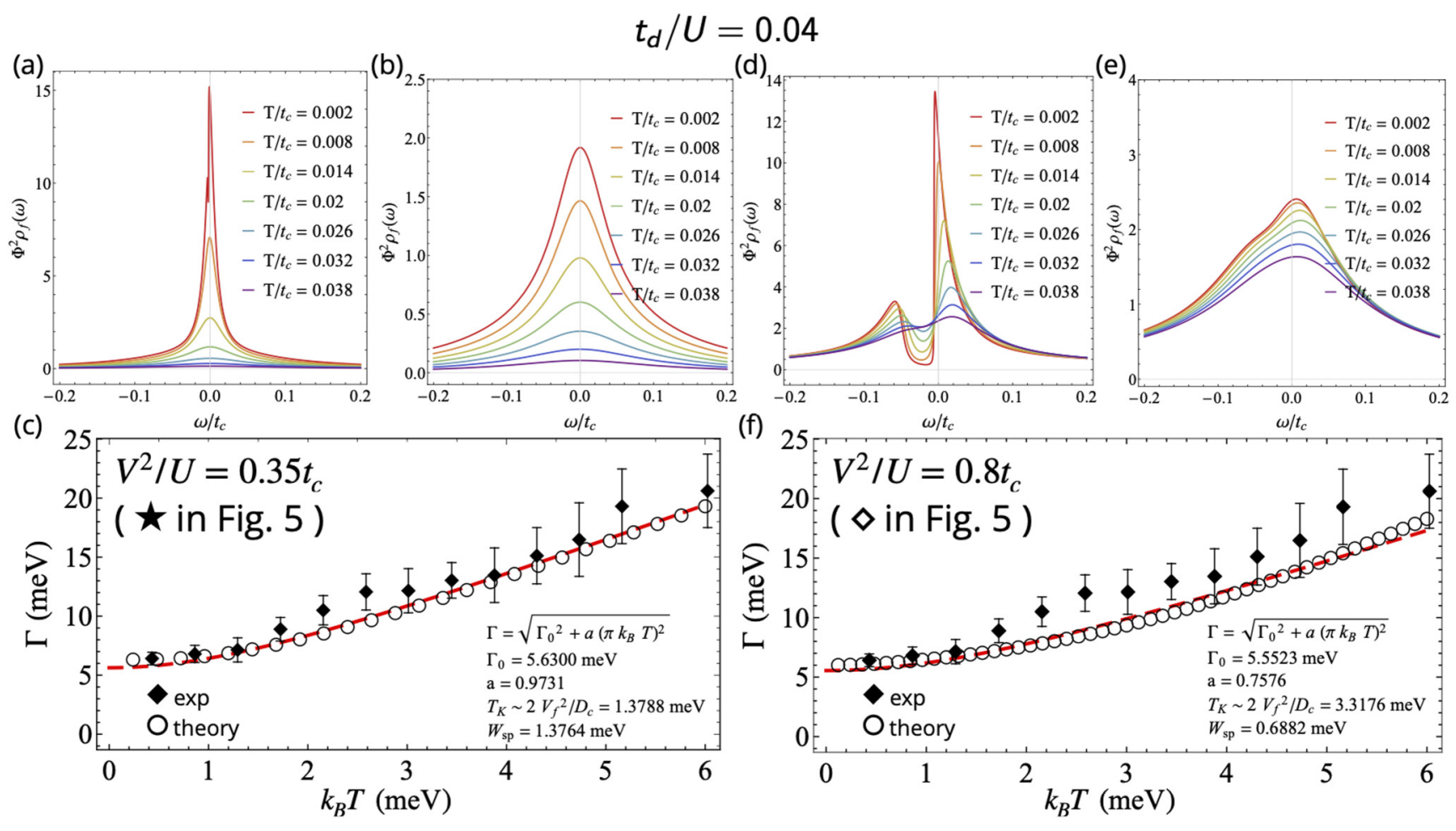

FIG. 12. LDOS at $t_{d} / U=0.04$. (a)-(c) With $V^{2} / U=0.35 t_{c}$ (indicated by $\star$ in Fig. 5). (a), (b) LDOS without/with $\gamma_{0}$ in the self-energy. (c) Width fitted to the experiment with $t_{c}=120 \mathrm{meV}$. The experimental data can be relatively well fitted by this case. (d) $-(\mathrm{f}) \mathrm{With} V^{2} / U=0.8 t_{c}$ (indicated by $\diamond$ in Fig. 5). (d), (e) LDOS without/with impurity scattering in the self-energy. (f) Fitting of the width to experiment with $t_{c}=60 \mathrm{meV}$. This case is much above the orange dashed line in Fig. 5 and the two quasiparticle bands are separated from each other.

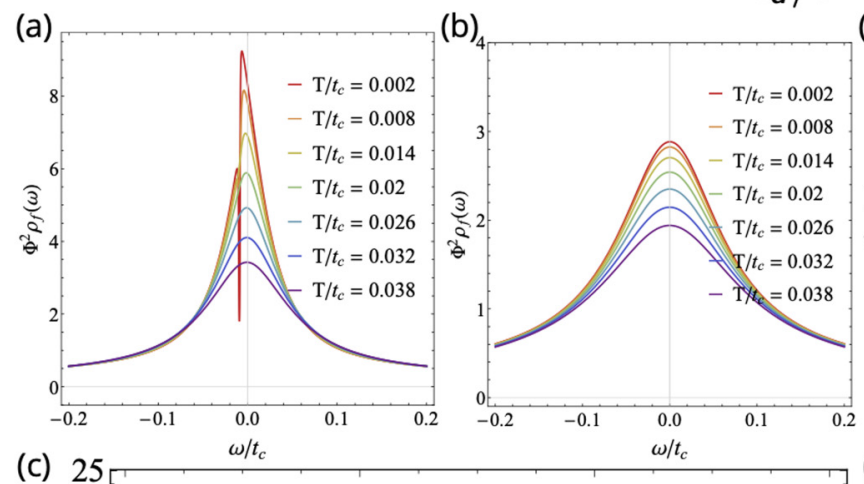

$$
t_{d} / U=0.08
$$
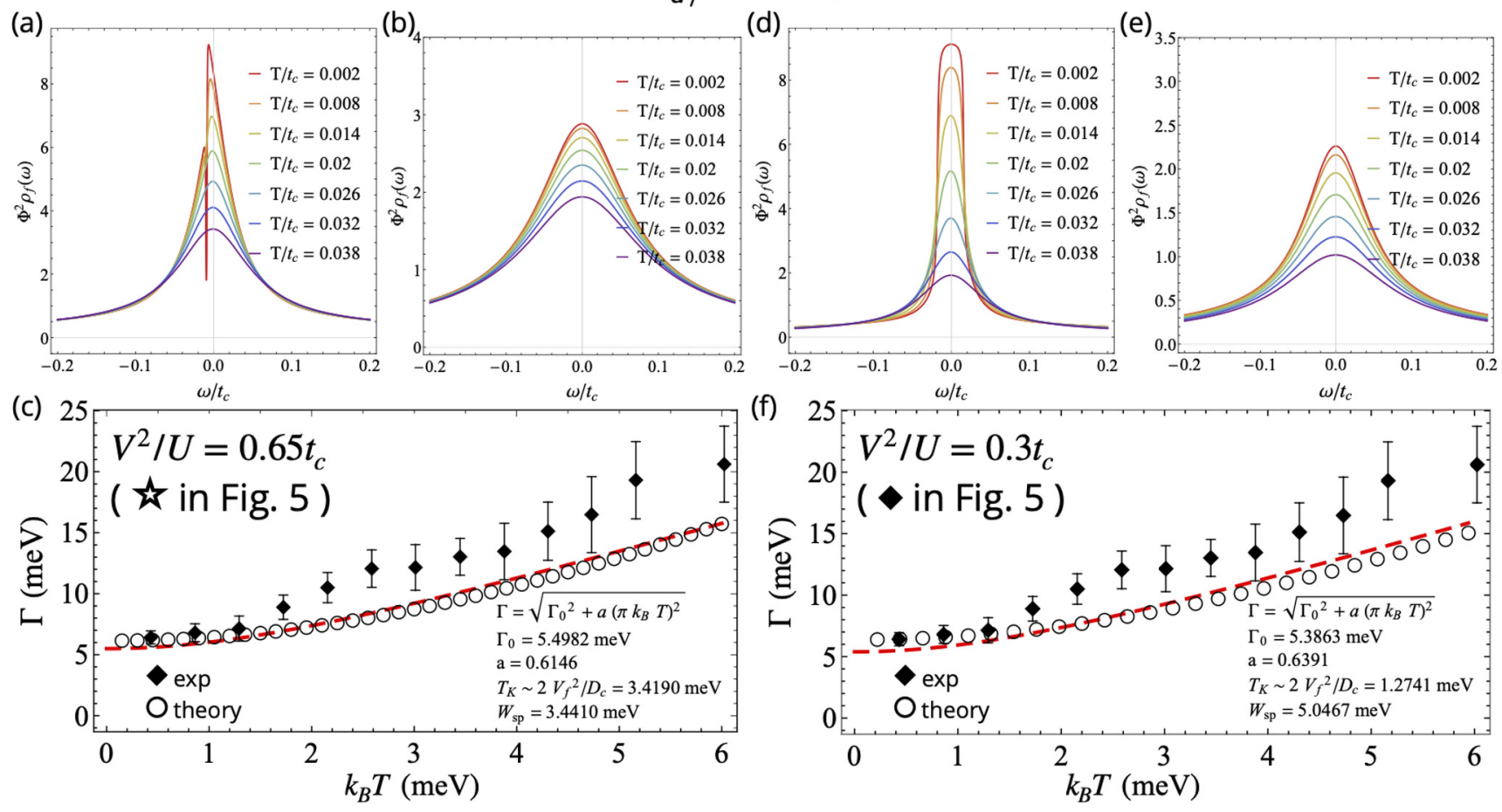

FIG. 13. LDOS at $t_{d} / U=0.08$. (a)-(c) With $V^{2} / U=0.65 t_{c}$ (indicated by 2 in Fig. 5). (a), (b) LDOS without/with $\gamma_{0}$ in the self-energy. (c) Fitting of the width to experiment with $t_{c}=75 \mathrm{meV}$. In this case the theory lies below the data because the slope $a$ is becoming too small. (d)-(f) With $V^{2} / U=0.3 t_{c}$ (indicated by in Fig. 5). (d), (e) LDOS without/with impurity scattering in the self-energy. (f) Fitting of the width to experiment with $t_{c}=110 \mathrm{meV}$. This case is below the orange dashed line and the two quasiparticle bands overlap. 
(a)
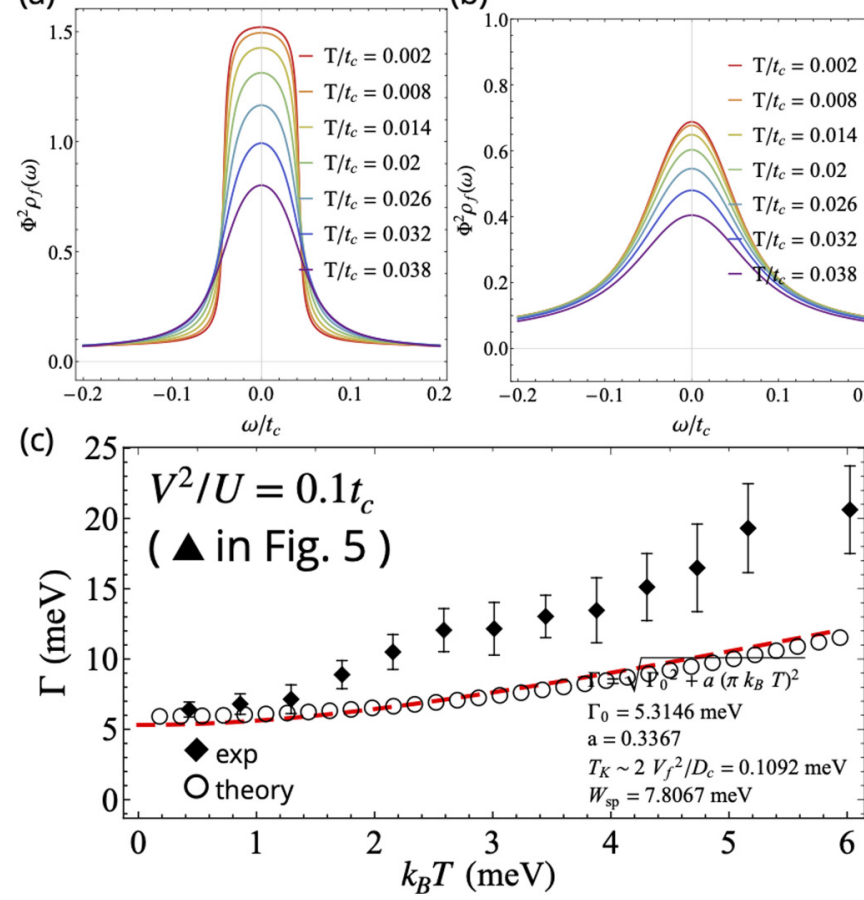

(b)

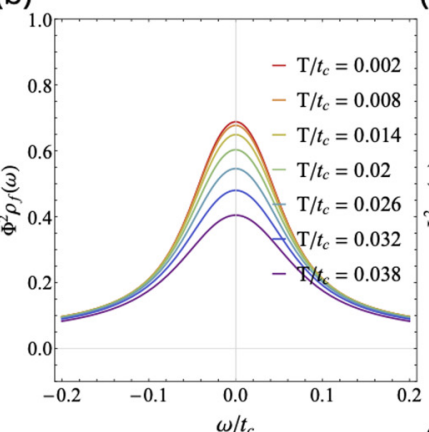

(d)

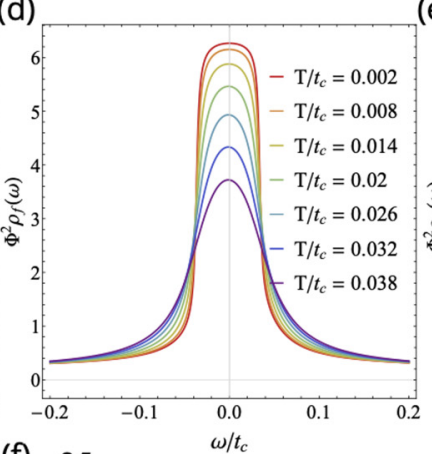

(e)

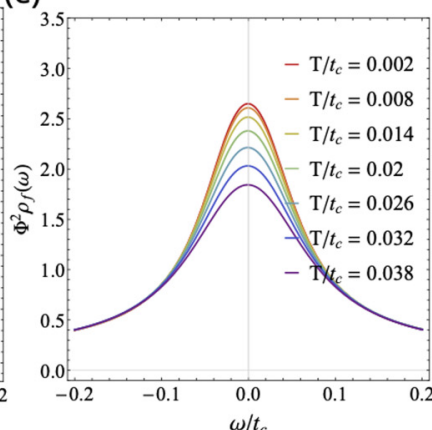

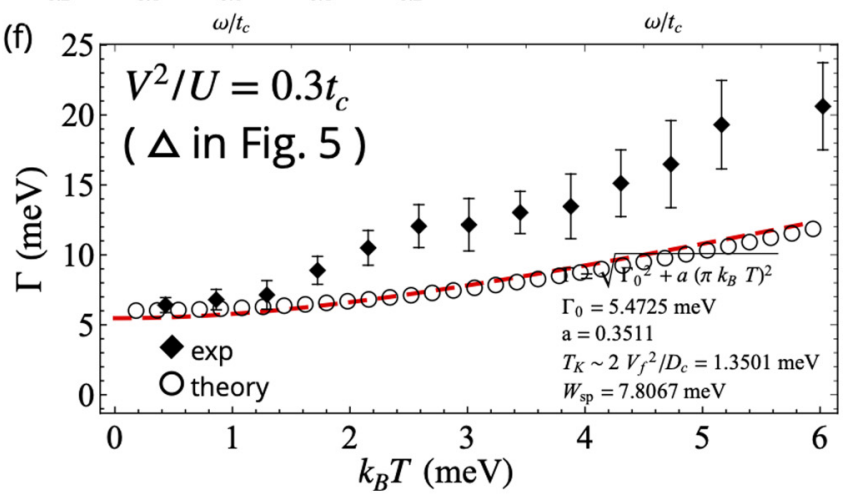

FIG. 14. LDOS at $t_{d} / U=0.11$. (a)-(c) With $V^{2} / U=0.1 t_{c}$ (indicated by $\boldsymbol{\Delta}$ in Fig. 5). (a), (b) LDOS without/with impurity scattering in the self-energy. (c) Fitting of the width to experiment with $t_{c}=90 \mathrm{meV}$. The slope of the theoretical data is too small to fit the experimental data. (d)-(f) With $V^{2} / U=0.3 t_{c}$ (indicated by $\triangle$ in Fig. 5). (d), (e) LDOS without/with impurity scattering in the self-energy. (f) Fitting of the width to experiment with $t_{c}=90 \mathrm{meV}$. Similar to the previous case, the slope of the theoretical data is too small to fit the experimental data.

between the theoretical and experimental results. $t_{c}$ is taken to be 120 and $75 \mathrm{meV}$ separately. We can see that the small spinon hopping case $t_{d} / U=0.04$ can give rise to a good fit to the experimental data. For the larger $t_{d}$ case $\left(t_{d} / U=0.08\right)$ the fit deteriorates because the coefficient $a$ is becoming too small.

We also checked cases with moderate $t_{d} / U$ but being farther away from the orange dashed line: $t_{d} / U=0.04, \quad V^{2} / U=0.8$ and $t_{d} / U=0.08, \quad V^{2} / U=0.3$ (indicated by $\diamond$ and $\downarrow$, respectively, in Fig. 5). Figures 12(d) and 12(e) show the LDOS for the first case without and with $\gamma_{0}$ included in the self-energy, and the LDOS for the latter case (without and with $\gamma_{0}$ in the self-energy) are presented in Figs. 13(d) and 13(e). The first case is above the orange dashed line in Fig. 5 with a large $J_{K}$, and the two quasiparticle bands are separated in energy. So the LDOS [Fig. 12(d)] has a gap sandwiched by two peaks. In the latter case, which is below the orange dashed line, the two quasiparticle bands overlap with each other and there is a flat peak in LDOS [see Fig. 13(d)]. Once $\gamma_{0}$ is introduced for both cases, the LDOS changes into a single-peak behavior for both cases [Figs. 12(e) and 13(e)]. The fittings of LDOS width to the experimental data for these two cases are shown in Figs. 12(f) and 13(f). One can see that while the parameter $a$ for $t_{d} / U=0.04$ still gives a reasonable fit, the value of $a$ for $t_{d} / U=0.08, V^{2} / U=0.3$ is too small and the width cannot be well fitted by Eq. (32). We conclude that as $t_{d} / U$ increases the fit deteriorates, especially away from the orange dashed line.
Finally, for large $t_{d} / U$ (here we take $t_{d} / U=0.11$ ) close to the critical value for the metal-insulator transition in the isolated Hubbard model, the LDOS for $V^{2} / U=0.1 t_{c}$ and $0.3 t_{c}$ (indicated by $\boldsymbol{\Delta}$ and $\Delta$ separately in Fig. 5) are shown in Figs. 14(a)-14(c) and 14(d)-14(f). As expected, the LDOS has a flat top near $\omega \sim 0$ without the inclusion of $\gamma_{0}$ in the self-energy [Figs. 14(a) and 14(d)], and will be broadened once $\gamma_{0}$ is introduced [Figs. 14(b) and 14(e)]. Figures 14(c) and 14(f) show the width for these cases and we see that the experimental data cannot be fitted by the theoretical results in this regime because the theoretical slope is too small.

To summarize, by including a Fermi-liquid type of (imaginary) self-energy into heavy quasiparticles' Green function, it is possible to obtain a single-peak behavior for the LDOS even in the Anderson limit. By modifying the value of $\gamma_{0}$, the width of LDOS can be well fitted by Eq. (32), which is the formula for a single impurity Kondo problem, as illustrated in Fig. 11(d). Moreover, adjusting $t_{c}$ to fit the experimental width value at the lowest temperature, our theory suggests that the experimental situation may be in or close to the Anderson limit of the model. On the other hand, for intermediate $t_{d} / U$ a reasonable fit can be obtained when the Kondo scale $J_{K}$ and the Heisenberg scale $J_{H}$ compete, resulting in a low-temperature width which is smaller than $J_{K}$ or $J_{H}$, as illustrated in Fig. 12(c). In addition, our theory predicts $a \sim 0.3$ if the hopping of the $d$ electrons is close to the critical value for the metal-insulator transition in the isolated Hubbard model, a value which does not fit the experimental data. 


\section{SUMMARY AND DISCUSSIONS}

We have studied a model of coupled correlated and itinerant electrons which naturally interpolates between the periodic Anderson model and the Hubbard model. Using a slave rotor mean-field approach we have obtained a phase diagram that summarizes the competition between a spinon Fermi-surface state weakly coupled to a metal and an interlayer coherent heavy Fermi-liquid metallic state (illustrated in Figs. 5, 6, and 8). In the localized or atomic limit where our model reduces to the periodic Anderson model, the Kondo coupling needed to destroy the spin liquid in favor of the metal, $J_{K} \sim V^{2} / U$, has a logarithmic dependence on the hopping of the correlated electrons in the putative spin liquid layer $t_{d} / U$, reflecting that the emergent scales determining the competition are the Kondo temperature $T_{K} \sim \rho^{-1} e^{-1 / J_{K} \rho}$ $\left(\rho \sim t_{c}^{-1}\right)$ and Heisenberg coupling $J_{H} \sim t_{d}^{2} / U$. Therefore, although technically in such limit the spin liquid is destabilized via a weak-coupling instability, the critical Kondo coupling needed to destabilize the spin liquid grows rather fast with the Heisenberg coupling, giving rise to the rapid rise of the boundary between the spin liquid and the heavy metal at small $t_{d} / U$ seen in Figs. 5, 6, and 8. In this limit one can use the measured saturation width $T_{K}$ to place an upper bound on the Heisenberg coupling $J_{H}$, resulting in a rather small bound of about $5 \mathrm{meV}$ from the experiments of [20]. On the other hand, at larger values of $t_{d} / U \sim 0.1$ when the spin liquid has a sizable bandwidth, the critical $J_{K}$ is comparable to $t_{d} / U$, and near the Mott transition the critical Kondo coupling needed to destabilize the spin liquid vanishes linearly with the distance of $t_{d} / U$ away from the critical value associated with the Mott metal-insulator transition, at mean-field level. However, we find that generically the peak width is dominated by the spinon bandwidth, leading to a width that is too broad and with too weak a temperature dependence to explain the data. The exception is when the system happens to fall near the crossover line indicated in orange in Fig. 5, where a reasonable fit to the data can also be obtained. In this case, the Kondo scale $J_{K}$ and the Heisenberg scale $J_{H}$ compete, giving rise to a narrow peak with a width which is smaller than either scale at low temperature. As a result, in this case the low-temperature width cannot be used as a bound for either scale, and it is possible that $J_{H}$ is much larger than the $5-\mathrm{meV}$ bound mentioned previously.

The above conclusion was reached by studying the LDOS of the heavy metal throughout this phase diagram, which can be directly accessed via STM experiments [20]. In the local moment periodic Anderson limit of the model the coherent hybridization of correlated and itinerant electrons in the heavy metal leads to the bare LDOS acquiring a two-peak structure due to the opening of a direct optical band gap. On the other hand, near the Mott metal-insulator transition the LDOS features a rather flat shape due to a relatively large spinon bandwidth. The measured LDOS is, however, further broadened by the intrinsic lifetime of the heavy quasiparticles arising from their interactions and also by disorder, leading to a smearing of the double-peak structure in the periodic Anderson model limit. We have argued that including these effects renders the double-peak structure effectively into a single peak, and we have found good agreement with the shape and temperature dependence of the peak reported in recent STM experiments [20], as illustrated in Fig. 11(d). We also find reasonable fit to the data at intermediate $t_{d} / U$ in the vicinity of the orange line in Fig. 5, as illustrated in Fig. 12(c).

We note that in the localized limit of small $t_{d} / U$ the Hubbard model in the triangular lattice is expected not to form a spinon Fermi-surface state, but to order into a conventional $120^{\circ} \mathrm{AFM}$ phase. This piece of physics is not captured in our slave rotor mean-field theory, which favors spin disordered ground states. Therefore, our results pose a challenge for the interpretation of the behavior of the standalone putative $1 \mathrm{~T}-\mathrm{TaSe}_{2}$ as a quantum spin liquid: if indeed the system is near the Anderson limit, this raises the possibility that it could be instead composed of localized moments that are rather weakly coupled and might ultimately weakly order at yet lower temperatures in cleaner samples. We, however, caution that we cannot definitely rule out that the putative spin liquid layer is at an intermediate coupling strength $t_{d} / U$ that brings the system closer to the Mott transition, where also a small interlayer tunneling can destabilize the spin liquid. An additional consideration is that the actual $1 \mathrm{~T}-\mathrm{TaSe}_{2}$ system involves multiple bands and is probably not described by a single band Mott-Hubbard model. While the spin liquid is stabilized only near the Mott transition in a single band model [25], it is possible that a multiband description can extend the spin liquid to lower effective $t_{d}$.

Additionally, to reiterate the potential uncertainties, we wish to note that the parameter $a$ in Eq. (32) that we used near the Mott transition has a Fermi-liquid form but it can be changed by tuning the value of $\gamma_{0}$ and $E_{0}$, which are, respectively, controlled by disorder and quasiparticle interactions, and hence are inherently difficult scales to estimate accurately.

We want also to point out that in our calculation, we considered the metallic electrons to have the same lattice constant and Brillouin zone as the correlated electrons. In doing so, we are imagining that in a more microscopic description one would be folding the Brillouin of the metallic $1 \mathrm{H}-\mathrm{TaSe}_{2}$, which does match with the smaller Brillouin zone of the star of David structure of $1 \mathrm{~T}-\mathrm{TaSe}_{2}$, and that after this one is only including one of the folded bands of itinerant electrons. However, the hybridization with electrons at higher-energy scales (coming from other folded bands) could also play an important role in determining the phase boundary and the form of LDOS, but such details lie beyond the scope of the considerations that we have explored in the present paper.

\section{ACKNOWLEDGMENTS}

We thank Michael F. Crommie, Wei Ruan, and Yi Chen for sharing their data and discussions. We also thank Peng Rao for fruitful discussions. P.A.L. acknowledges support by the U.S. Department of Energy Office of Basic Sciences Grant No. DE-FG02-03ER46076.

\section{APPENDIX A: FINITE TEMPERATURE ROTOR MEAN-FIELD APPROACH}

As mentioned in the main text, for the order parameter of the metallic phase, $\Phi=\left\langle e^{i \theta}\right\rangle$, we estimate its value by taking the average with respect to a single site 
Hamiltonian:

$$
\begin{aligned}
H_{\theta}^{(1)} & =-K_{\theta}\left(e^{i \theta}+e^{-i \theta}\right)+\frac{U}{2} n_{\theta}^{2} \\
& =H_{K}+H_{U},
\end{aligned}
$$

where $H_{K}=-K_{\theta}\left(e^{i \theta}+e^{-i \theta}\right)$ and $H_{U}=\frac{U}{2} n_{\theta}^{2}$. We have taken $\lambda=0$ to fulfill the constraint Eq. (3) and the half filling of the spinon. Because we are interested in the large $U$ limit of the model $\left(t_{d} / U \lesssim 1 / 8\right)$, it is reasonable to use a first-order perturbation (in $H_{K}$ ) to estimate the expectation value:

$$
\begin{aligned}
\left\langle e^{i \theta}\right\rangle & =\frac{\operatorname{Tr}\left(e^{-\beta\left(H_{U}+H_{K}\right)} e^{i \theta}\right)}{\operatorname{Tr}\left(e^{-\beta\left(H_{U}+H_{K}\right)}\right)} \\
& \approx-\int_{0}^{\beta} d \tau \operatorname{Tr}\left(e^{-\beta H_{U}} e^{\tau H_{U}} H_{K} e^{-\tau H_{U}} e^{i \theta}\right) / \operatorname{Tr}\left(e^{-\beta H_{U}}\right),
\end{aligned}
$$

where one can take the trace with the eigenbasis of angular momentum $n_{\theta},\{|n\rangle\}$, which satisfies $n_{\theta}|m\rangle=m|m\rangle$ and $e^{i \theta}|n\rangle=|n+1\rangle$, and we will denote the eigenvalue of $H_{U}$ by $E_{n}=\frac{U}{2} n^{2}$. It is straightforward to obtain

$$
\begin{aligned}
& -\int_{0}^{\beta} d \tau \operatorname{Tr}\left(e^{-\beta H_{U}} e^{\tau H_{U}} H_{K} e^{-\tau H_{U}} e^{i \theta}\right) \\
& =K_{\theta} \sum_{n} \int_{0}^{\beta} d \tau e^{-\beta E_{n}} e^{-\beta E_{n}} e^{\tau\left(E_{n}-E_{n+1}\right)} \\
& =K_{\theta} \sum_{n} \frac{e^{-\beta E_{n+1}}-e^{-\beta E_{n}}}{E_{n}-E_{n+1}} \\
& \operatorname{Tr}\left(e^{-\beta H_{U}}\right)=\sum_{n} e^{-\beta E_{n}}
\end{aligned}
$$

so one finally arrives at

$$
\begin{gathered}
\left\langle e^{i \theta}\right\rangle \approx \chi_{\theta, 1} K_{\theta}, \\
\chi_{\theta, 1}=\sum_{n} \frac{e^{-\beta E_{n+1}}-e^{-\beta E_{n}}}{E_{n}-E_{n+1}} / \sum_{n} e^{-\beta E_{n}} .
\end{gathered}
$$

By taking the zero-temperature limit, one can recover the zero-temperature result given by

$$
\lim _{\beta \rightarrow \infty} \chi_{\theta, 1}(\beta)=4 / U \text {. }
$$

Next, we extrapolate the expression above, which is valid only for small $K_{\theta}$, with the phenomenological formula

$$
\left\langle e^{i \theta}\right\rangle=\frac{K_{\theta}}{\sqrt{\chi_{\theta, 1}^{-2}+K_{\theta}^{2}}},
$$

which recovers the behavior from Eq. (A5) at small $K_{\theta}$ and also the approach of $\left\langle e^{i \theta}\right\rangle \rightarrow 1$, which is expected at large $K_{\theta}$ (it is also consistent with the constraint that $\left\langle e^{i \theta}\right\rangle \leqslant 1$ ).

For $\left\langle e^{-i \theta_{i}} e^{i \theta_{j}}\right\rangle$, one can perform same kind of calculation. We estimate it by taking the expectation value with respect to the Hamiltonian:

$$
\tilde{H}_{\theta}=\frac{U}{2} \sum_{i} n_{\theta, i}^{2}-2 T_{\theta} \sum_{\langle i, j\rangle}\left(e^{-i \theta_{i}} e^{i \theta_{j}}+\text { H.c. }\right),
$$

and taking the $T_{\theta}$ term as a perturbation, after some algebra, one obtains that

$$
\begin{gathered}
\left\langle e^{-i \theta_{i}} e^{i \theta_{j}}\right\rangle \approx \chi_{\theta, 2} T_{\theta}, \\
\chi_{\theta, 2}=2\left(\sum_{n_{i} \neq n_{j}+1} \frac{e^{-\beta\left(E_{n_{i}-1}+E_{n_{j}+1}\right)}-e^{-\beta\left(E_{n_{i}}+E_{n_{j}}\right)}}{E_{n_{i}}+E_{n_{j}}-\left(E_{n_{i}-1}+E_{n_{j}+1}\right)}\right. \\
\left.+\sum_{n} \beta e^{-\beta\left(E_{n}+E_{n-1}\right)}\right) /\left(\sum_{n} e^{-\beta E_{n}}\right)^{2},
\end{gathered}
$$

and for the zero-temperature limit one recovers the value $\chi_{\theta, 2}=4 / U$. Because we are interested in the small $t_{d}$ limit (remember that $T_{\theta}=t_{d} \chi_{0}$ ), we simply use Eq. (A10) throughout our calculations.

It should be noted that the current mean field would predict an artificial second-order phase transition for any low-temperature phase with finite $\left\langle e^{i \theta}\right\rangle$ to a high-temperature phase with $\left\langle e^{i \theta}\right\rangle=0$, similar to the case of slave boson descriptions at mean-field level [32]. In reality, there is no such phase transition as a function of temperature but only a crossover [37,38], and the expectation value of $\left\langle e^{i \theta}\right\rangle$ is always finite at nonzero temperatures. However, the zero-temperature transitions which are the focus of the main paper are allowed to be sharp second-order phase transitions in principle $[39,40]$.

\section{APPENDIX B: TUNNELING DOS OF THE SINGLE IMPURITY ANDERSON MODEL}

In this section, we briefly review the theory of tunneling DOS for a single impurity Anderson model and give a more thorough derivation on the fitting of STM results expanding on the previous studies by Nagaoka et al. [29].

For a single impurity Anderson model, one can calculate the tunneling DOS of the local electron using perturbation theory since there is no phase transition as the on-site interaction $U$ increases [23]. Early theoretical calculations [35,36] showed that the (retarded) Green function of the local electron for the particle-hole symmetric case reads (valid at small $\omega$ and $T$ )

$$
\begin{aligned}
G_{d}(\omega, T) & =\frac{1}{\omega-\epsilon_{d}-\operatorname{Re} \Sigma(\omega)+i \Delta-i \operatorname{Im} \Sigma(\omega, T)} \\
& =\frac{Z}{\omega-\tilde{\epsilon}_{d}+i Z[\Delta-\operatorname{Im} \Sigma(\omega, T)]},
\end{aligned}
$$

where

$$
\begin{aligned}
\tilde{\epsilon}_{d} & =\epsilon_{d}+\operatorname{Re} \Sigma(0) \approx 0, \\
\operatorname{Im} \Sigma(\omega, T) & =-\frac{\Delta}{2} \alpha^{2}\left[\left(\frac{\omega}{T_{K}}\right)^{2}+\pi^{2}\left(\frac{T}{T_{K}}\right)^{2}\right],
\end{aligned}
$$

where $\alpha$ is a number of order unity and equals $\pi / 4$. In the second line of Eq. (B1) we follow standard practice and expand $\operatorname{Re} \Sigma(\omega)$ to linear order in $\omega$ near the pole with

$$
Z=\frac{1}{1-\left.\partial_{\omega} \operatorname{Re} \Sigma(\omega)\right|_{\omega=0}}=\frac{T_{K}}{\alpha \Delta} .
$$


Then it is straightforward to obtain the spectral function:

$$
\begin{aligned}
\rho_{d}(\omega) & =\frac{Z^{2}}{\pi} \frac{[\Delta-\operatorname{Im} \Sigma(\omega)]}{\omega^{2}+Z^{2}[\Delta-\operatorname{Im} \Sigma(\omega)]^{2}} \\
& =\frac{Z^{2} \Delta}{\pi} \frac{1+\frac{1}{2} \alpha^{2}\left(\frac{\omega^{2}}{T_{K}^{2}}+\frac{\pi^{2} T^{2}}{T_{K}^{2}}\right)}{\omega^{2}+Z^{2} \Delta^{2}\left[1+\frac{1}{2} \alpha^{2}\left(\frac{\omega^{2}}{T_{K}^{2}}+\frac{\pi^{2} T^{2}}{T_{K}^{2}}\right)\right]^{2}} \\
& =\frac{1}{\pi \Delta} \frac{1}{\frac{\omega^{2} /(Z \Delta)^{2}}{1+\frac{1}{2} \alpha^{2}\left(\frac{\omega^{2}}{T_{K}^{2}}+\frac{\pi^{2} T^{2}}{T_{K}^{2}}\right)}+1+\frac{1}{2} \alpha^{2}\left(\frac{\omega^{2}}{T_{K}^{2}}+\frac{\pi^{2} T^{2}}{T_{K}^{2}}\right)} \\
& =\frac{1}{\pi \Delta} \frac{1}{\frac{\alpha^{2} \omega^{2} / T_{K}^{2}}{1+\frac{1}{2} \alpha^{2}\left(\frac{\omega^{2}}{T_{K}^{2}}+\frac{\pi^{2} T^{2}}{T_{K}^{2}}\right)}+1+\frac{1}{2} \alpha^{2}\left(\frac{\omega^{2}}{T_{K}^{2}}+\frac{\pi^{2} T^{2}}{T_{K}^{2}}\right)} .
\end{aligned}
$$

In the previous work by Nagaoka et al. [29], they did not include the expansion near the pole, which amounts to setting $Z=1$. With this and setting $\alpha=1$, they argued that the $\omega$ term in the denominator of Eq. (B1) can be dropped and they arrive at the incorrect result that $\rho_{d} \propto 1 / \operatorname{Im} \Sigma(\omega, T)$, i.e.,

$$
\rho_{d}(\omega)=\frac{1}{\pi \Delta} \frac{1}{1+\frac{1}{2}\left(\frac{\omega^{2}}{T_{K}^{2}}+\frac{\pi^{2} T^{2}}{T_{K}^{2}}\right)}
$$

with the prediction that the width reads

$$
\Gamma_{\exp }=\sqrt{2 T_{K}^{2}+\pi^{2} T^{2}},
$$

which suggests the slope of $\Gamma$ with respect to $T$ is approximately $\pi$ for $T \gg T_{K}$

However, as we can see from the second line in Eq. (B1), due to the fact that $Z \approx T_{K} / \Delta \ll 1, \omega$ cannot be dropped. This is seen explicitly in Eq. (B4), where the first term in the denominator dropped by Nagaoka et al. [29] is clearly of the same order as the rest and should be kept. Nevertheless, we shall show below that the conclusion that the slope of $\Gamma$ with respect to $T$ is approximately $\pi$ at relatively high temperature is actually valid. The more complete Eq. (B4) implies that the line shape is not a simple Lorentzian. Instead, we calculate the half width at half height by requiring $\Gamma$ to satisfy $\rho_{d}(\Gamma)=\rho_{d}(0) / 2$, which leads to

$$
\alpha^{2}\left(\frac{\Gamma}{T_{K}}\right)^{2}=\left[1+\frac{1}{2} \alpha^{2}\left(\frac{\pi T}{T_{K}}\right)^{2}\right]^{2}-\left[\frac{1}{2} \alpha^{2}\left(\frac{\Gamma}{T_{K}}\right)^{2}\right]^{2},
$$

and after some algebra one can show that

$$
\Gamma=\frac{\sqrt{2}}{\alpha}\left[\sqrt{T_{K}^{4}+\left(T_{K}^{2}+\frac{1}{2} \alpha^{2} \pi^{2} T^{2}\right)^{2}}-T_{K}^{2}\right]^{1 / 2} .
$$

In the low-temperature limit, the width can be approximated as

$$
\Gamma \approx \sqrt{\frac{2(\sqrt{2}-1)}{\alpha^{2}} T_{K}^{2}+\frac{1}{\sqrt{2}} \pi^{2} T^{2}},
$$

but on the other hand, for large $T$ such that $T \gg T_{K}, \Gamma$ can be approximated as

$$
\Gamma \approx \pi T
$$

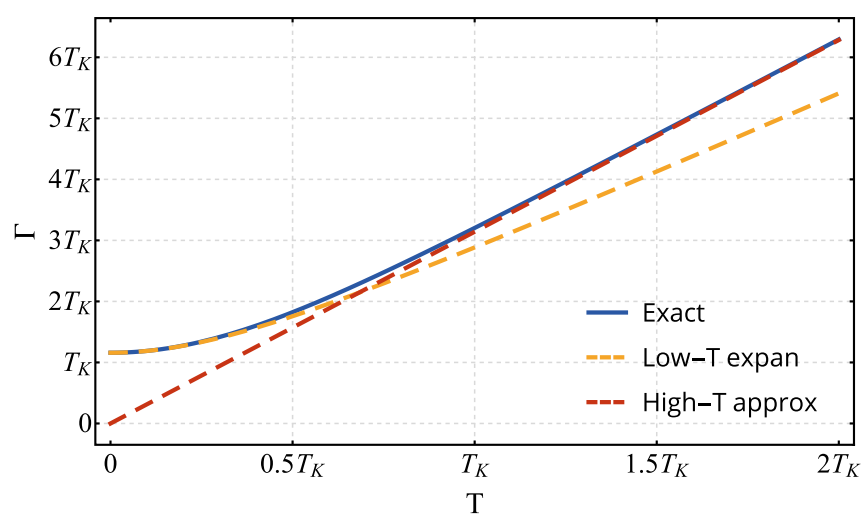

FIG. 15. Plot of the LDOS width $\Gamma$ with respect to the temperature $T$ based on Fermi-liquid theory. The blue solid line stands for the exact result Eq. (B8), the orange dashed line indicates the low-temperature expanded form Eq. (B9), and the red dashed line shows the high-temperature approximated form Eq. (B10).

Going back to Eq. (B4), we see that in the large $T$ limit the first term in the denominator becomes a nonzero constant. It affects the effective definition of the zero-temperature width in terms of $T_{K}$, but does not affect the high-temperature limit of the linewidth. Although the low-temperature expansion Eq. (B9) seems to suggest that the slope of the $\Gamma-T$ curve would saturate to $\pi / 2^{1 / 4}$ at relatively large temperatures (see the orange dashed line in Fig. 15), the slope derived from Eq. (B8) actually saturates to $\pi$ at higher temperatures, as indicated by the blue line in Fig. 15.

According to Eq. (B8), the zero-temperature width should be $\Gamma(T=0)=[2(\sqrt{2}-1)]^{\frac{1}{2}} / \alpha \approx 1.16 T_{K}$, while Eq. (B6) predicts $\Gamma_{\exp }(T=0)=\sqrt{2} T_{K}$. Therefore, with a given set of experimental data of $\Gamma$ versus $T$, the extracted $T_{K}$ using Eq. (B6) would be slightly smaller than the one predicted from Eq. (B8). On the other hand, both expressions suggest that $\Gamma$ has an approximately linear dependence on $T$ for $T \sim T_{K}$ with a $\pi$ slope.

Finally, comparing the Fermi-liquid theory presented above and the more exact numerical renormalization-group (NRG) calculation, one can see that both theories imply that the LDOS is not a simple Lorentzian form. The Fermi-liquid theory suggests $\Gamma(T=0) \approx 1.16 T_{K}$ while the NRG suggests $\Gamma(T=0)=T_{K}$. The NRG LDOS curve can be quantitatively well fitted by a phenomenological expression suggested by Frota and Oliveira [41] and Frota [42]:

$$
\begin{aligned}
\rho_{f}(\omega) & =\frac{2}{\pi \Gamma_{A}} \operatorname{Re}\left[\left(\frac{\omega+i \Gamma_{K}}{i \Gamma_{K}}\right)^{-1 / 2}\right] \\
& =\frac{2}{\pi \Gamma_{A}}\left(\frac{1+\sqrt{1+\left(\omega / \Gamma_{K}\right)^{2}}}{2\left[1+\left(\omega / \Gamma_{K}\right)^{2}\right]}\right)^{1 / 2},
\end{aligned}
$$

with $\Gamma_{A}$ and $\Gamma_{K}$ being fitting parameters. However, it should be noted that this formula is a phenomenological parametrization of the model and is not able to predict the temperature dependence of LDOS and its width [42]. 
[1] P. Anderson, MRS Bull. 8, 153 (1973).

[2] P. Fazekas and P. W. Anderson, Philos. Mag. 30, 423 (1974).

[3] P. W. Anderson, Science 235, 1196 (1987).

[4] C. Broholm, R. J. Cava, S. A. Kivelson, D. G. Nocera, M. R. Norman, and T. Senthil, Science 367, eaay0668 (2020).

[5] L. Savary and L. Balents, Rep. Prog. Phys. 80, 016502 (2016).

[6] Y. Zhou, K. Kanoda, and T.-K. Ng, Rev. Mod. Phys. 89, 025003 (2017).

[7] K. T. Law and P. A. Lee, Proc. Natl. Acad. Sci. USA 114, 6996 (2017).

[8] W.-Y. He, X. Y. Xu, G. Chen, K. T. Law, and P. A. Lee, Phys. Rev. Lett. 121, 046401 (2018).

[9] K. Rossnagel, J. Phys. Condens. Matter 23, 213001 (2011).

[10] M. Kratochvilova, A. D. Hillier, A. R. Wildes, L. Wang, S.-W. Cheong, and J.-G. Park, npj Quantum Mater. 2, 42 (2017).

[11] M. Klanjšek, A. Zorko, R. Žitko, J. Mravlje, Z. Jagličić, P. K. Biswas, P. Prelovšek, D. Mihailovic, and D. Arčon, Nat. Phys. 13, 1130 (2017).

[12] E. Tosatti and P. Fazekas, J. Phys. Colloques 37, C4-165 (1976).

[13] J. Wilson, F. D. Salvo, and S. Mahajan, Adv. Phys. 24, 117 (1975).

[14] A. Ribak, I. Silber, C. Baines, K. Chashka, Z. Salman, Y. Dagan, and A. Kanigel, Phys. Rev. B 96, 195131 (2017).

[15] Y. J. Yu, Y. Xu, L. P. He, M. Kratochvilova, Y. Y. Huang, J. M. Ni, L. Wang, S.-W. Cheong, J.-G. Park, and S. Y. Li, Phys. Rev. B 96, 081111(R) (2017).

[16] H. Murayama, Y. Sato, T. Taniguchi, R. Kurihara, X. Z. Xing, W. Huang, S. Kasahara, Y. Kasahara, I. Kimchi, M. Yoshida, Y. Iwasa, Y. Mizukami, T. Shibauchi, M. Konczykowski, and Y. Matsuda, Phys. Rev. Research 2, 013099 (2020).

[17] K. Rossnagel and N. V. Smith, Phys. Rev. B 73, 073106 (2006).

[18] F. Di Salvo, R. Maines, J. Waszczak, and R. Schwall, Solid State Commun. 14, 497 (1974).

[19] Y. Chen, W. Ruan, M. Wu, S. Tang, H. Ryu, H.-Z. Tsai, R. Lee, S. Kahn, F. Liou, C. Jia, O. R. Albertini, H. Xiong, T. Jia, Z. Liu, J. A. Sobota, A. Y. Liu, J. E. Moore, Z.-X. Shen, S. G. Louie, S.-K. Mo, and M. F. Crommie, Nat. Phys. 16, 218 (2020).
[20] W. Ruan, Y. Chen, S. Tang, J. Hwang, H.-Z. Tsai, R. Lee, M. Wu, H. Ryu, S. Kahn, F. Liou, C. Jia, A. Aikawa, C. Hwang, F. Wang, Y. Choi, S. G. Louie, P. A. Lee, Z.-X. Shen, S.-K. Mo, and M. F. Crommie, arXiv:2009.07379.

[21] S. Doniach, Physica B+C 91, 231 (1977).

[22] A. C. Hewson, The Kondo Problem to Heavy Fermions, Cambridge Studies in Magnetism (Cambridge University, Cambridge, England, 1993).

[23] P. Coleman, Introduction to Many-Body Physics (Cambridge University, Cambridge, England, 2015).

[24] S. Florens and A. Georges, Phys. Rev. B 70, 035114 (2004).

[25] S.-S. Lee and P. A. Lee, Phys. Rev. Lett. 95, 036403 (2005).

[26] O. I. Motrunich, Phys. Rev. B 72, 045105 (2005).

[27] V. Sunko, F. Mazzola, S. Kitamura, S. Khim, P. Kushwaha, O. J. Clark, M. D. Watson, I. Marković, D. Biswas, L. Pourovskii, T. K. Kim, T.-L. Lee, P. K. Thakur, H. Rosner, A. Georges, R. Moessner, T. Oka, A. P. Mackenzie, and P. D. C. King, Sci. Adv. 6, eaaz0611 (2020).

[28] E. Zhao and A. Paramekanti, Phys. Rev. B 76, 195101 (2007).

[29] K. Nagaoka, T. Jamneala, M. Grobis, and M. F. Crommie, Phys. Rev. Lett. 88, 077205 (2002).

[30] N. Read and D. M. Newns, J. Phys. C: Solid State Phys. 16, 3273 (1983).

[31] N. Read and D. M. Newns, J. Phys. C: Solid State Phys. 16, L1055 (1983).

[32] P. Coleman, Phys. Rev. B 29, 3035 (1984).

[33] A. Auerbach and K. Levin, Phys. Rev. B 34, 3524 (1986).

[34] L. Zheng and S. Das Sarma, Phys. Rev. B 53, 9964 (1996).

[35] K. Yamada, Prog. Theor. Exp. Phys. 53, 970 (1975).

[36] T. A. Costi, A. C. Hewson, and V. Zlatic, J. Phys. Condens. Matter 6, 2519 (1994).

[37] P. Coleman, J. Magn. Magn. Mater. 47-48, 323 (1985).

[38] R. Franco, M. S. Figueira, and M. E. Foglio, Phys. Rev. B 66, 045112 (2002).

[39] T. Senthil, M. Vojta, and S. Sachdev, Phys. Rev. B 69, 035111 (2004).

[40] T. Senthil, Phys. Rev. B 78, 045109 (2008).

[41] H. O. Frota and L. N. Oliveira, Phys. Rev. B 33, 7871 (1986).

[42] H. O. Frota, Phys. Rev. B 45, 1096 (1992). 